\title{
Multistep Wind Speed Forecasting Using a Novel Model Hybridizing Singular Spectrum Analysis, Modified Intelligent Optimization, and Rolling Elman Neural Network
}

\author{
Zhongshan Yang and Jian Wang \\ School of Statistics, Dongbei University of Finance and Economics, Dalian, China \\ Correspondence should be addressed to Jian Wang; jianwang0826@163.com
}

Received 27 April 2016; Revised 16 September 2016; Accepted 26 September 2016

Academic Editor: Mario Cools

Copyright (C) 2016 Z. Yang and J. Wang. This is an open access article distributed under the Creative Commons Attribution License, which permits unrestricted use, distribution, and reproduction in any medium, provided the original work is properly cited.

Wind speed high-accuracy forecasting, an important part of the electrical system monitoring and control, is of the essence to protect the safety of wind power utilization. However, the wind speed signals are always intermittent and intrinsic complexity; therefore, it is difficult to forecast them accurately. Many traditional wind speed forecasting studies have focused on single models, which leads to poor prediction accuracy. In this paper, a new hybrid model is proposed to overcome the shortcoming of single models by combining singular spectrum analysis, modified intelligent optimization, and the rolling Elman neural network. In this model, except for the multiple seasonal patterns used to reduce interferences from the original data, the rolling model is utilized to forecast the multistep wind speed. To verify the forecasting ability of the proposed hybrid model, $10 \mathrm{~min}$ and $60 \mathrm{~min}$ wind speed data from the province of Shandong, China, were proposed in this paper as the case study. Compared to the other models, the proposed hybrid model forecasts the wind speed with higher accuracy.

\section{Introduction}

In the past few decades, with environmental degradation and resource depletion, renewable energy [1] has received more attention. Wind energy, one of the cleanest forms of renewable energy, is developing rapidly throughout the world. With the rapid increase in the utilization of wind energy, the primary concern is the security and stability of feeding electricity into the grid [2]. Wind speed highaccuracy forecasting is an important part of the electrical system monitoring and control. However, due to the instability of wind energy and the inherent complexity, transferring electricity into the power grid is limited and costly $[3,4]$. To improve the efficiency of wind power and reduce the comprehensive cost of wind energy, accurate prediction of wind speed is necessary.

Many methods have been proposed to improve the forecasting accuracy of wind speed in recent decades. Based on the computational mechanism, these forecasting models can be grouped into four main categories: (i) physical models, (ii) statistical models, (iii) intelligence models, and (iv) hybrid forecasting models [5].

Physical methods $[6,7]$, which are based on the lower atmosphere or numerical weather prediction (NWP), can accurately forecast the wind speed. However, the physical methods require long running times and are not applicable for short-term forecasting. Statistical models [8-11], which are known as time-series-based models, do not apply historical data. These models are trained with measurement data and the differences between forecasted and actual wind speed are used to adjust the model parameters. The ARMA and the ARIMA models are the most popular models used to forecast future wind speed. Many forecasting results based on statistical models show that these models are useful in the wind speed forecasting fields [12-15]. These models have numerous advantages, and the approaches need only historical wind speed data and are easy to implement. However, if the nonlinear characteristics of the wind speed series are prominent, the prediction accuracy of these methods decreases rapidly. The intelligent methods adopt artificial intelligence 
(AI) theories or evolutionary algorithms to forecast wind speed. Many intelligent methods are used to forecast wind speed, such as ANN (Artificial Neural Network) [16-18], FLM (Fuzzy Logic Method) [19, 20], and SVM (Support Vector Machine) [18, 21-26]. Unlike the single methods, the hybrid methods proposed by experts and scholars always combine several models to improve the accuracy of wind speed forecasting. Most of the recently proposed forecasting methods are hybrids, and the decomposition algorithms are often used to enhance their precision [27, 28].

Chaotic theory has been used to handle time series in many fields [29-31]. Considering chaotic characteristics of the wind speed series, a hybrid prediction model was introduced in [32] using largest Lyapunov exponent prediction method to predict. Due to the inherent complexity of wind speed, describing the moving trend of wind speed and accurate prediction is difficult. Therefore, many studies use other methods to enhance the forecasting capacity of the original series. The specific methods include hybrid models that employ different approaches or combine different forecasting models to extract the inner traits of the original series in different aspects to perform wind speed forecasting. For the former type of hybrid models, the most common methods, such as wavelet transform (WT), singular spectrum analysis (SSA), and empirical mode decomposition (EMD), are used to preprocess the original series and forecast the wind speeds [32-35]. These data processing methods are used to eliminate the influence of outliers on the forecasting accuracy, thereby improving the forecasting accuracy.

In this paper, a novel algorithm is proposed that hybridizes SSA (singular spectrum analysis), FAPSO (Firefly Algorithm and Particle Swarm Optimization), and RENN (rolling Elman neural network), to forecast wind speed. To verify the performance of the model, several hybrid models and single models are also used to forecast wind speed. In this model, besides the multiple seasonal patterns used to reduce interferences from the original data, the rolling model is utilized to forecast the multistep wind speed. To verify the forecasting ability of the proposed hybrid model, $10 \mathrm{~min}$ and $60 \mathrm{~min}$ wind speed data from the province of Shandong, China, were used as the case study.

The details of the algorithm are described below, and the flow diagram is shown in Figure 1.

Step 1. The SSA is used to decompose the original wind speed datasets into several subseries. Then, the new series is reconstructed. The wind speed data used in this paper is typically a chaotic time series, and the use of SSA can eliminate the influence of outliers and improve the prediction accuracy of the wind speed forecast model.

Step 2. The hybrid optimization algorithm (FAPSO) that combines the FA with the PSO is utilized to optimize the weights and thresholds of the ENN model. The optimization algorithm can provide better initial weights and thresholds to the ENN and improve the search ability. Compared with the single optimization model, the hybrid optimization model has better optimization effects.
Step 3. Construct the ENN model for the reconstructed series. Then, use the established model to forecast the onestep wind speed. The optimized ENN model can avoid getting trapped into local optimum and the global searching ability of the algorithm is enhanced.

Step 4. The rolling ENN model is used to forecast the multistep results. Multistep wind speed forecasting with high-precision is helpful for electricity production to produce various benefits, such as avoiding a power-grid collapse, reducing production costs, and reducing the spinning reserve capacity of thermal power units.

Step 5. The Diebold-Mariano test is used to validate the accuracy and stability of the proposed model.

\section{Methodology}

In this paper, numerous methods are involved. In this section, the relative algorithms including singular spectrum analysis, the firefly algorithm, particle swarm optimization, and the hybrid model are described in detail.

2.1. Singular Spectrum Analysis. The singular spectrum analysis [36-38] is a signal processing technique capable of capturing the intrinsic oscillation modes of a signal. The SSA has two main stages: decomposition and reconstruction.

2.1.1. Wind Speed Time Series Decomposition. The decomposition has two main steps: embedding and SVD (singular value decomposition). During the embedding stage, given a time series $X_{N}=\left(x_{0}, \ldots, x_{N-1}\right), N$ is the effective length. Define $L(1<L<N)$ called "window length" and $K=$ $N-L+1$.

To perform the embedding, the original time series is mapped into a sequence of lagged vectors of size $L$ by forming $K=N-L+1$ lagged vectors

$$
\mathbf{x}_{i}=\left(x_{i-1}, \ldots, x_{i+L-2}\right)^{T}, \quad i=1, \ldots, K .
$$

Then, the trajectory matrix is derived:

$$
\begin{aligned}
\mathbf{X} & =\left[\mathbf{x}_{i}: \cdots: \mathbf{x}_{K}\right]=\left(x_{i j}\right)_{i, j=1}^{L, K} \\
& =\left(\begin{array}{cccc}
x_{0} & x_{1} & \cdots & x_{K-1} \\
x_{1} & x_{2} & \cdots & x_{K} \\
\vdots & \vdots & \ddots & \vdots \\
x_{L-1} & x_{L} & \cdots & x_{N-1}
\end{array}\right) .
\end{aligned}
$$

From the trajectory matrix, both the rows and columns of $\mathbf{X}$ are subseries of the original series, and $\mathbf{X}$ is a Hankel matrix, which means it has equal elements on antidiagonals.

In the singular value decomposition step, the singular value decomposition of the matrix $\mathbf{X}$ can be computed through eigenvalues and eigenvectors of the matrix $\mathbf{X X}^{T}$. Suppose $\lambda_{i}\left(\lambda_{1}>\lambda_{2}>\cdots>\lambda_{L}>0\right)$ as the eigenvalues of $\mathbf{X X}^{T}$, and suppose $U_{i}$ as the corresponding eigenvector. 
The singular value decomposition of the trajectory matrix $\mathbf{X}$ is shown below:

$$
\begin{aligned}
\mathbf{X} & =\mathbf{X}_{1}+\mathbf{X}_{2}+\cdots+\mathbf{X}_{d}, \\
V_{i} & =\frac{\mathbf{X}^{T} U_{i}}{\sqrt{\lambda_{i}}} \\
\mathbf{X}_{i} & =U_{i} V_{i} \sqrt{\lambda_{i}} .
\end{aligned}
$$

The matrices $\mathbf{X}_{i}$ are elementary matrices. The collection $\left(U_{i}, V_{i}^{T}, \sqrt{\lambda_{i}}\right)$ is called the $i$ th eigentriple of the SVD. Each eigentriple consists of an eigenvector, a factor vector, and a singular value. $U_{i}$ and $V_{i}$ are $i$ th left singular vectors and right singular vectors of $\mathbf{X}$, respectively.

2.1.2. Wind Speed Series Reconstruction. The decomposition has two main steps: grouping and diagonal averaging. During the grouping stage, $I=\{1,2, \ldots, d\}$ is split into $m$ disjoint groups $I_{1}, I_{2}, \ldots, I_{m}$. Let $I=\left\{i_{1}, i_{2}, \ldots, i_{k}\right\}$; then, the resultant matrix $\mathbf{X}_{I}$ corresponding to the group $I$ is defined as

$$
\mathbf{X}_{I}=\mathbf{X}_{i 1}+\mathbf{X}_{i 2}+\cdots+\mathbf{X}_{i K} .
$$

These matrices are computed for $I=I_{1}, I_{2}, \ldots, I_{m}$ and the expansion of (3) leads to the decomposition as follows:

$$
\mathbf{X}=\mathbf{X}_{I 1}+\mathbf{X}_{I 2}+\cdots+\mathbf{X}_{I m} .
$$

In the diagonal averaging step, the grouped decomposition in (6) will be transformed into a new series of length $N$. Let $Y$ be a $L \times K$ matrix with elements $y_{i j}(1 \leq i \leq L, 1 \leq j \leq$ $K)$. Diagonal averaging transfers the matrix $Y$ to the series $h_{1}, h_{2}, \ldots, h_{m}$ by the following formula:

$$
\mathbf{h}_{k}= \begin{cases}\frac{1}{k+1} \sum_{m=1}^{k+1} y_{m, K-m+2}^{*}, & 0 \leq k \leq \mathbf{l}^{*}-1 \\ \frac{1}{k^{*}} \sum_{m=1}^{\mathbf{l}^{*}} y_{m, K-m+2}^{*}, & \mathbf{l}^{*}-1 \leq k \leq \mathbf{k}^{*} \\ \frac{1}{N-k} \sum_{m=k-\mathbf{k}^{*}+2}^{N-\mathbf{k}^{*}+1} y_{m, K-m+2}^{*}, & \mathbf{k}^{*} \leq k \leq N,\end{cases}
$$

where $\mathbf{l}^{*}=\min (L, K) ; \mathbf{k}^{*}=\max (L, K) ; N=L+K-1$; if $L<K, y_{i j}^{*}=y_{i j}$; and if not $y_{i j}^{*}=y_{j i}$.

The original time series can be decomposed into $m$ series by SSA

$$
x_{n}=\sum_{k=1}^{m} \widetilde{x}_{n}^{(k)}, \quad n=0,1, \ldots, N-1,
$$

where $\widetilde{x}_{n}^{(k)}$ is the result of diagonal averaging of the matrix $\mathbf{X}_{I k}$.

2.2. Elman Neural Network (ENN). The Elman recurrent neural network, proposed by Elman, is a partial recurrent network model [39]. Compared with classic feed-forward perception and pure recurrent network, ENN has a context layer that feeds back the hidden layer outputs in the previous time-steps. The context layer can enhance the ability of processing dynamic information and improve the forecasting accuracy.

The neurons contained in each layer are used to disseminate information from one layer to another. The nonlinear state space expression of Elman networks is as follows:

$$
\begin{aligned}
\mathbf{y}(k) & =g\left(\omega^{3} \mathbf{x}(k)\right), \\
\mathbf{x}(k) & =f\left(\omega^{1} \mathbf{x}_{c}(k)+\omega^{2}(\mathbf{u}(k-1))\right), \\
\mathbf{x}_{c}(k) & =\mathbf{x}(k-1),
\end{aligned}
$$

where $\mathbf{y}$ is the $m$-dimension output node vector; $\mathbf{x}$ is $n$ dimension hidden layer node vector; $\mathbf{u}$ is $r$-dimension input layer vector; $\mathbf{x}_{c}$ is the feedback state vector; $\omega^{1}, \omega^{2}$, and $\omega^{3}$ denote their corresponding weights; $g(*)$ is the transfer function of the output neuron; and $f(*)$ is the transfer function of the hidden neuron.

Then, adjust the weights of the network to minimize the squared error between the actual values and forecasting results:

$$
\min \mathbf{E}=\sum_{k=1}^{n}\left(y_{k}(\omega)-\tilde{y}_{k}(\omega)\right)^{2},
$$

where $\tilde{y}_{k}(\omega)$ is a target output vector.

Although the ENN has strong predictive power, the limitations are obvious. The initial weights and threshold values of ENN are randomly generated, the training speed is slow, and ENN is susceptible to falling into the local optimal value. The intelligent optimization algorithm can effectively overcome these shortcomings.

\subsection{Firefly Algorithm and Particle Swarm Optimization} (FAPSO). The optimization algorithm is composed of the firefly algorithm and particle swarm optimization. Compared with a single optimization algorithm, the proposed optimization algorithm avoids many shortcomings and determines a better solution.

2.3.1. Firefly Algorithm (FA). The firefly algorithm, proposed by Yang, is a multimodal nature inspired metaheuristic algorithm based on the flashing behavior of fireflies [40, 41]. The algorithm has proved effective in solving linear design problems and multimodal optimization problems.

The firefly algorithm has two stages, which are described as follows.

Step 1. The brightness is dependent on the intensity of light emitted by the firefly. Suppose there are a group of fireflies and the position for an $i$ th firefly is $\boldsymbol{\xi}_{i}$, where $f\left(\xi_{i}\right)$ indicates the fitness value of the firefly. The brightness $I$ of a firefly is chosen to reflect the fitness value of its current position

$$
I_{i}=f\left(\xi_{i}\right) \quad \text { where } 1 \leq i \leq n .
$$

Step 2. All fireflies have a unique attractiveness $I_{i}$, which indicates the ability to attract other fireflies. The attractiveness 


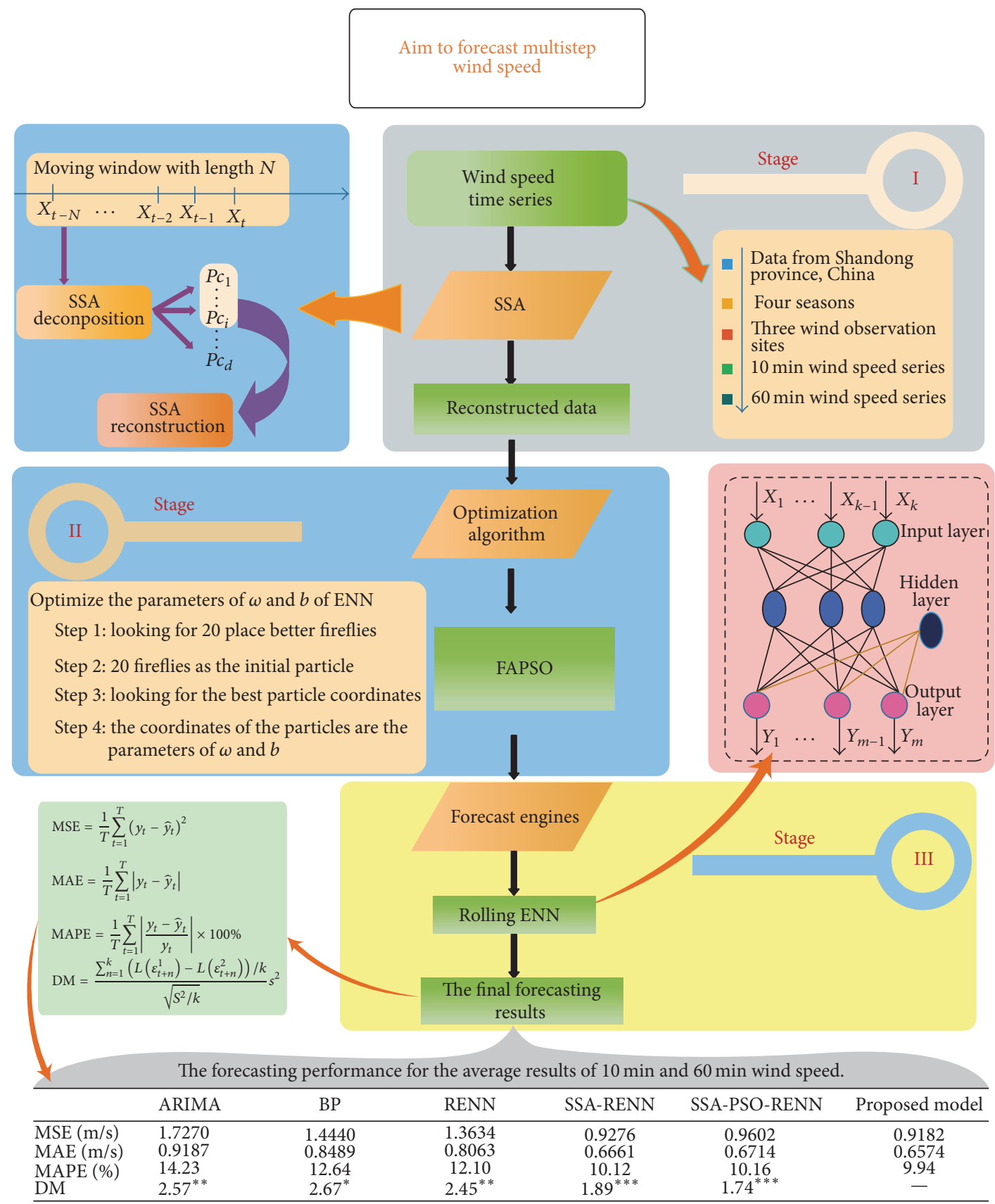

$*$ is $1 \%$ significance level; $* *$ is $5 \%$ significance level; $* * *$ is $10 \%$ significance level.

FIGURE 1: The flowchart of the proposed integrated forecasting model.

is related to the distance factor $r_{i j}$ at locations $\mathbf{x}_{i}$ and $\mathbf{x}_{j}$, and between the two corresponding fireflies, $i$ and $j$ are given by

$$
r_{i j}=\left\|\xi_{i}-\xi_{j}\right\|=\sqrt{\sum_{k=1}^{d}\left(\xi_{i, k}-\xi_{j, k}\right)^{2}} .
$$
as

The attractiveness function $\beta(r)$ of the firefly is computed

$$
\beta_{i j}\left(r_{i j}\right)=\beta_{0} e^{-\gamma r_{i j}^{2}}
$$

where $\beta_{0}$ is the largest attraction and $\gamma$ is the coefficient of light absorption.

The movement of the less bright firefly toward the most bright firefly is computed as

$$
\begin{aligned}
\xi_{i}(t+1)= & \xi_{i}(t)+\beta_{0} e^{-\gamma r_{i j}{ }^{2}}\left(\xi_{j}-\xi_{i}\right) \\
& +\eta\left(\text { rand }-\frac{1}{2}\right),
\end{aligned}
$$

where $\eta$ is the randomization parameter and rand is a randomly selected number between the interval $[0,1]$. A rudimentary FA algorithm is outlined in Algorithm 1. 


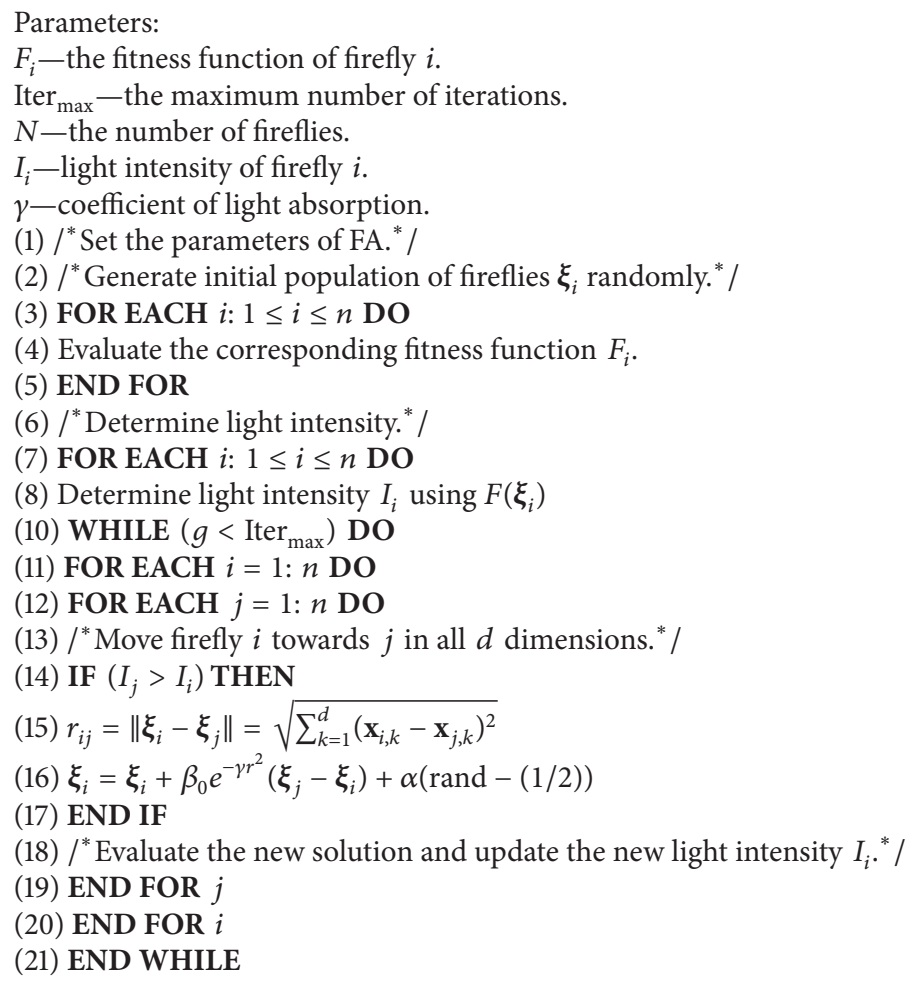

Algorithm 1: FA.

2.3.2. Particle Swarm Optimization (PSO). Particle Swarm Optimization (developed by Kennedy and Eberhart) [42, 43] is based on the behavior of birds. The principle of PSO is assuming a location has no mass or volume, flying like a bird in multidimensional space, and not only adjusts its position but also exchanges information about its current position in search space according to its own earlier experience and that of its neighbors. In this mechanism, members of a swarm communicate their information and modify their positions and velocities using the group information according to the best position appearing in the current movement of the swarm. The particles of the swarm find the optimal point by cooperation. The particle velocity and position updating formulas are shown below:

$$
\begin{aligned}
v_{i}(t+1)= & \omega * v_{i}(t)+c_{1} * \operatorname{rand}(1) \\
& *\left(\text { lbest }_{i}(t)-x_{i}(t)\right)+c_{2} * \operatorname{rand}(1) \\
& *\left(\text { gbest }_{i}(t)-x_{i}(t)\right), \\
x_{i}(t+1)= & x_{i}(t)+v_{i}(t+1),
\end{aligned}
$$

where $\omega$ is the inertia weight; $v_{i}$ is the velocity of the $i$ th particle; $c_{1}$ and $c_{2}$ are acceleration coefficients (nonnegative constant); $\operatorname{rand}(1)$ is a random value between 0 and $1 ; x_{i}(t)$ is the position of the $i$ th particle; lbest $(t)$ and gbest $(t)$ denote local best particle of the $i$ th particle and global best particle among local bests at time $t$.

A rudimentary PSO algorithm isoutlined in Algorithm 2.
2.3.3. Hybrid Optimization Algorithm. In this section, a modified optimization model hybridizing firefly algorithm and particle swarm optimization is proposed to improve the accuracy of wind speed forecasting. The specifics of the FAPSO are described below.

Step 1. The firefly algorithm was used to optimize $n$ fireflies.

(1.1) Generate initial population of fireflies randomly.

(1.2) Compute the brightness of each firefly by using objective function.

(1.3) Move firefly and evaluate new fireflies.

(1.4) Rank the fireflies and find the current best as the firefly researched.

(1.5) Optimize $n$ fireflies in total.

Step 2. The particle swarm algorithm is used to search the best particle.

(2.1) The fireflies, searched in the first step, are the initial population.

(2.2) Calculate the fitness values of particles.

(2.3) Find the global best position.

(2.4) Update the positions and velocities of the agents.

(2.5) Determine whether the termination conditions have been satisfied; if so, proceed to step (2.1) and continue to search; otherwise, continue to step (2.6).

(2.6) Find the best global position. 
Parameters:

$n$-the number of particles.

Iter $_{\max }$ - the maximum number of iterations.

$w, c, v$ - the parameters of PSO.

(1) $/{ }^{*}$ Set the parameters of PSO.* /

(2) $/{ }^{*}$ Generate initial population of particles randomly. ${ }^{*} /$

(3) FOR EACH $i: 1 \leq i \leq n$ DO

(4) $x_{i}=\operatorname{rand}()$;

(5) $\mathbf{P}=\left\{x_{i}^{\text {iter }}: 1 \leq i \leq n\right\}$;

(6) END FOR

(7) $/{ }^{*}$ Find the best value of particles. ${ }^{*} /$

(8) WHILE $\left(g \leq\right.$ Iter $\left._{\max }\right)$ DO

(9) $/{ }^{*}$ Find the best fitness value for each candidates. ${ }^{*} /$

(10) FOR EACH $i: x_{i}^{\text {iter }} \in \mathbf{P}$ DO

(11) Calculate each particle fitness function.

(12) IF $\left(\right.$ pbest $\left._{i}>\operatorname{fitness}\left(x_{i}^{\text {iter }}\right)\right)$ THEN

(13) pbest $_{i}=\operatorname{fitness}\left(x_{i}^{\text {iter }}\right)$

(14) END IF

(15) END FOR

(16) $/{ }^{*}$ Choose the candidate with the best fitness value of all the candidates ${ }^{*} /$

(17) FOR EACH $i: x_{i}^{\text {iter }} \in \mathbf{P}$ DO

(18) IF ( gbest $^{>}$pbest $\left._{i}\right)$ THEN

(19) gbest $^{=}$pbest $_{i}$

(20) $x_{\text {best }}=x_{i}^{\text {iter }}$

(21) END IF

(22) END FOR

(23) $/{ }^{*}$ Update the values of all the candidates by using PSO's evolution equations.* $/$

(24) FOR EACH $i: x_{i}^{\text {iter }} \in \mathbf{P}$ DO

(25) $v_{i}(t+1)=\omega * v_{i}(t)+c_{1} * \operatorname{rand}() *\left(\right.$ lbest $\left._{i}(t)-x_{i}(t)\right)+c_{2} * \operatorname{rand}() *\left(\right.$ gbest $\left._{i}(t)-x_{i}(t)\right)$

(26) $x_{i}(t+1)=x_{i}(t)+v_{i}(t+1)$

(27) END FOR

(28) END WHILE

Algorithm 2: PSO.

2.3.4. Test of FAPSO. To verify the optimization performance and convergence speed of the modified algorithm, four benchmark functions are selected in this paper. These benchmark functions have different characteristics, which are used to fully investigate the optimization ability of the algorithm. The four common test functions are shown in Table 1. The experimental parameters of PSO and FAPSO are shown in Table 2.

Thirty experiments, searching for the minimum value point by 2000 iterations, were carried out independently. The results, including the maximum, minimum, average, and standard deviations, are displayed in Table 3. The results show that the optimization effect of FAPSO is better than PSO.

2.4. The Proposed Hybrid Model. In this paper, a novel algorithm, SSA-FAPSO-RENN, is proposed to forecast wind speed. SSA is used to acquire the moving tendency of wind speed and enhance the forecasting abilities. The hybrid optimization algorithm (FAPSO) that combines the FA and the PSO is utilized to optimize the parameters of the ENN model. To forecast the multistep wind speed, the rolling Elman neural network (RENN) model is used.
For the convenience of narrative, the proposed hybrid model is named the SSA-FAPSO-RENN model.

\section{Experimental Simulation}

In this section, the details of experimental simulation will be introduced. Wind speed series of $10 \mathrm{~min}$ and $60 \mathrm{~min}$ are used to verify the effect of the model.

3.1. Performance Metric. The primary concern is to determine whether the prediction model is superior to other models. The performance of the model is usually evaluated using statistical criterions.

To estimate the forecasting performance, the DieboldMariano (DM) test and three error criterions are adopted, including MAE, MAPE, and MSE. DM test [44] is a comparison test that focuses on the predictive accuracy and can be used to evaluate the forecasting performance of the proposed hybrid model and other comparing models. The details of DM test are given as follows:

$$
\mathrm{DM}=\frac{\sum_{n=1}^{k}\left(L\left(\varepsilon_{t+n}^{1}\right)-L\left(\varepsilon_{t+n}^{2}\right)\right) / k}{\sqrt{S^{2} / k}} s^{2},
$$


TABLE 1

\begin{tabular}{lcclc}
\hline Function name & \multicolumn{1}{c}{ Test function } & Variable domain & Global optimum & Function characteristic \\
\hline Sphere & $f_{1}(X)=\sum_{i=1}^{D} x_{i}^{2}$ & $x_{i} \in[-5.12,5.12]$ & $x_{i}=0, f_{1}(X)=0$ & Unimodal \\
Rosenbrock & $f_{2}(X)=\sum_{i=1}^{D-1}\left[100\left(x_{i}^{2}-x_{i+1}\right)^{2}+\left(x_{i}-1\right)^{2}\right]$ & $x_{i} \in[-2.084,2.084]$ & $x_{i}=0, f_{2}(X)=0$ & Unimodal \\
Rastrigin & $f_{3}(X)=\sum_{i=1}^{D}\left(x_{i}^{2}-10 \cos \left(2 \pi x_{i}\right)+10\right)$ & $x_{i} \in[-5.12,5.12]$ & $x_{i}=0, f_{3}(X)=0$ & Multimodal \\
Griewank & $f_{4}(X)=\frac{1}{4000} \sum_{i=1}^{D} x_{i}^{2}-\prod_{i=1}^{D} \cos \left(\frac{x_{i}}{\sqrt{i}}\right)+1$ & $x_{i} \in[-5.12,5.12]$ & $x_{i}=0, f_{4}(X)=0$ & Multimodal \\
\hline
\end{tabular}

TABLE 2: The experimental parameters of PSO and FAPSO.

\begin{tabular}{lcc}
\hline Experimental parameters & PSO & FAPSO \\
\hline Maximum iteration & 2000 & 2000 \\
Population size & 40 & 40 \\
$w$ & 0.729 & 0.729 \\
$c$ & 1.494 & 1.494 \\
$v_{\max }$ & 0.5 & 0.5 \\
Dimension & 30 & 30 \\
Number of experiments & 30 & 30 \\
\hline
\end{tabular}

where $L(*)$ is the loss function. $\varepsilon_{t+n}^{1}$ and $\varepsilon_{t+n}^{2}$ are the forecast errors from two models. $S^{2}$ is an estimator of the variance of $d_{n}=L\left(\varepsilon_{t+n}^{1}\right)-L\left(\varepsilon_{t+n}^{2}\right)$. The hypothesis test is defined as

$$
\begin{aligned}
& H_{0}: E\left(d_{n}\right)=0, \\
& H_{1}: E\left(d_{n}\right) \neq 0 .
\end{aligned}
$$

The null hypothesis is that the two forecasts have the same accuracy. Under the null hypothesis, the test statistics DM are asymptotically $N(0,1)$ distributed. If $|\mathrm{DM}|>z_{\alpha / 2}$, the null hypothesis will be rejected.

The detailed equations of these three error criterions are given as follows.

MSE (Mean Squared Error)

$$
\text { MSE }=\frac{1}{T} \sum_{t=1}^{T}\left(y_{t}-\widehat{y}_{t}\right)^{2} .
$$

MAE (Mean Absolute Error)

$$
\text { MAE }=\frac{1}{T} \sum_{t=1}^{T}\left|y_{t}-\widehat{y}_{t}\right| .
$$

MAPE (Mean Absolute Percentage Error)

$$
\text { MAPE }=\frac{1}{T} \sum_{t=1}^{T}\left|\frac{y_{t}-\widehat{y}_{t}}{y_{t}}\right| \times 100 \%,
$$

where $y_{t}$ and $\hat{y}_{t}$ denote the real and predicted values at time $t$, respectively.
3.2. Wind Speed Datasets. To verify the forecasting ability of the proposed hybrid model, $10 \mathrm{~min}$ and $60 \mathrm{~min}$ wind speed data (January 1, 2011, to November 9, 2011) from the province of Shandong, China, are proposed as the case study in this paper. In the two tests, multiple seasonal patterns are used to reduce interferences from the original data, March 1 to May 31 (spring), June 1 to August 31 (summer), September 1 to November 9 (fall), and January 1 to February 28 (winter), and the wind speed datasets are randomly selected.

To further assess the forecasting accuracy, every wind speed series is divided into a training set and a validation set. In addition, an entire day of data will be used as a test set to test the forecasting ability of the models.

The first case study is $10 \mathrm{~min}$ wind speed forecasting. The total number of available samples is 1152 . The training set also includes 806 wind speed datasets and the validation set includes 140 wind speed datasets. The remaining data are used to calculate the predictive ability of these models. Figure 2 shows four wind speed datasets from three wind observation sites corresponding to the four seasons.

The second case study is $60 \mathrm{~min}$ wind speed forecasting. The total number of available samples is 1032 . The training set includes 806 wind speed datasets, and the validation set includes 202 wind speed datasets, and the remaining data are the test set. Figure 3 shows four wind speed datasets $(60 \mathrm{~min}$ wind speed) from three wind observation sites corresponding to the four seasons.

From Figures 2 and 3, several features can be summarized:

(a) The data for four seasons are quite different.

(b) There are three wind observation sites. The wind speed data from the same site is similar.

(c) The intensity of the wind in winter is large but small for the wind in summer.

(d) The experimental datasets reveal the chaotic nature and intrinsic complexity of wind speed.

3.3. Set Parameters of the Related Models. In this paper, a novel algorithm that hybridizes SSA, FAPSO, and RENN is proposed to forecast wind speed. Parameters have a large influence on prediction accuracy.

3.3.1. SSA Decomposition and Reconstruction. SSA performs well with complex irregular time series. The parameter 
TABLE 3: Test results of PSO and FAPSO.

\begin{tabular}{lccccc}
\hline Test function & Algorithm & Min value & Max value & Average value & Standard deviation \\
\hline \multirow{2}{*}{ Sphere } & PSO & 0.4276 & 1.198 & 0.7030 & 0.1740 \\
& FAPSO & 0.0481 & 0.1158 & 0.0837 & 0.0156 \\
Rosenbrock & PSO & 56.2745 & 131.5162 & 90.7276 & 18.7055 \\
& FAPSO & 35.1140 & 43.7005 & 38.3808 & 1.8540 \\
Rastrigin & PSO & 169.0 & 252.1567 & 201.6199 & 21.5151 \\
& FAPSO & 40.8482 & 71.1049 & 59.012 & 6.5202 \\
Griewank & PSO & 0.0478 & 0.3839 & 0.0884 & 0.0607 \\
& FAPSO & 0.0090 & 0.0200 & 0.0145 & 0.0026 \\
\hline
\end{tabular}
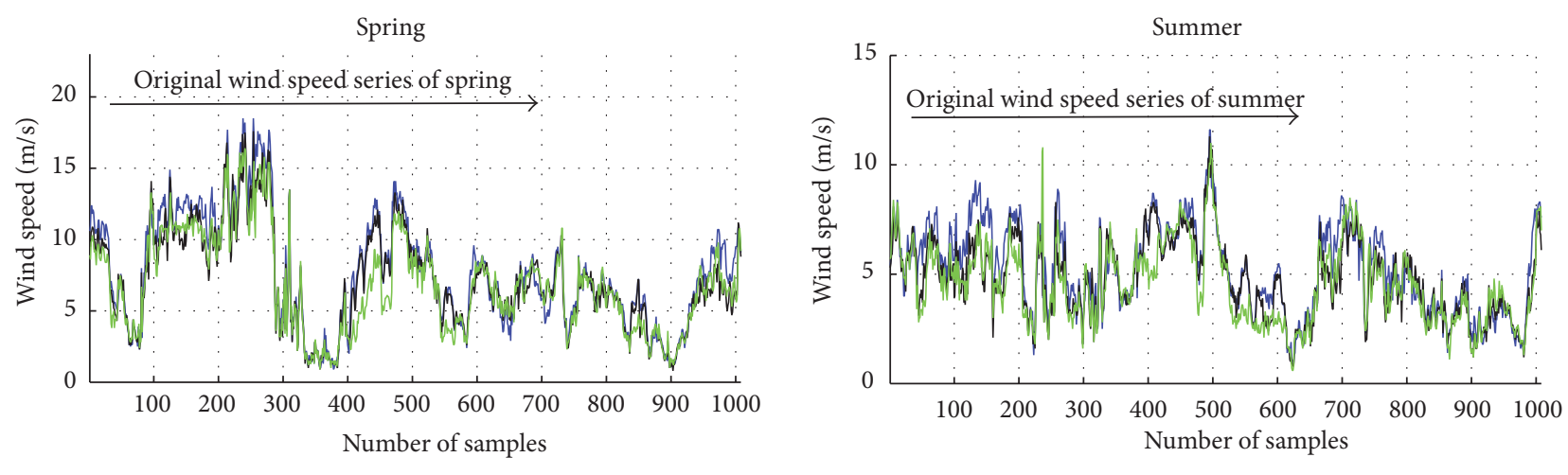

Datasets from wind observation site A
D Datasets from wind observation site B
Datasets from wind observation site C
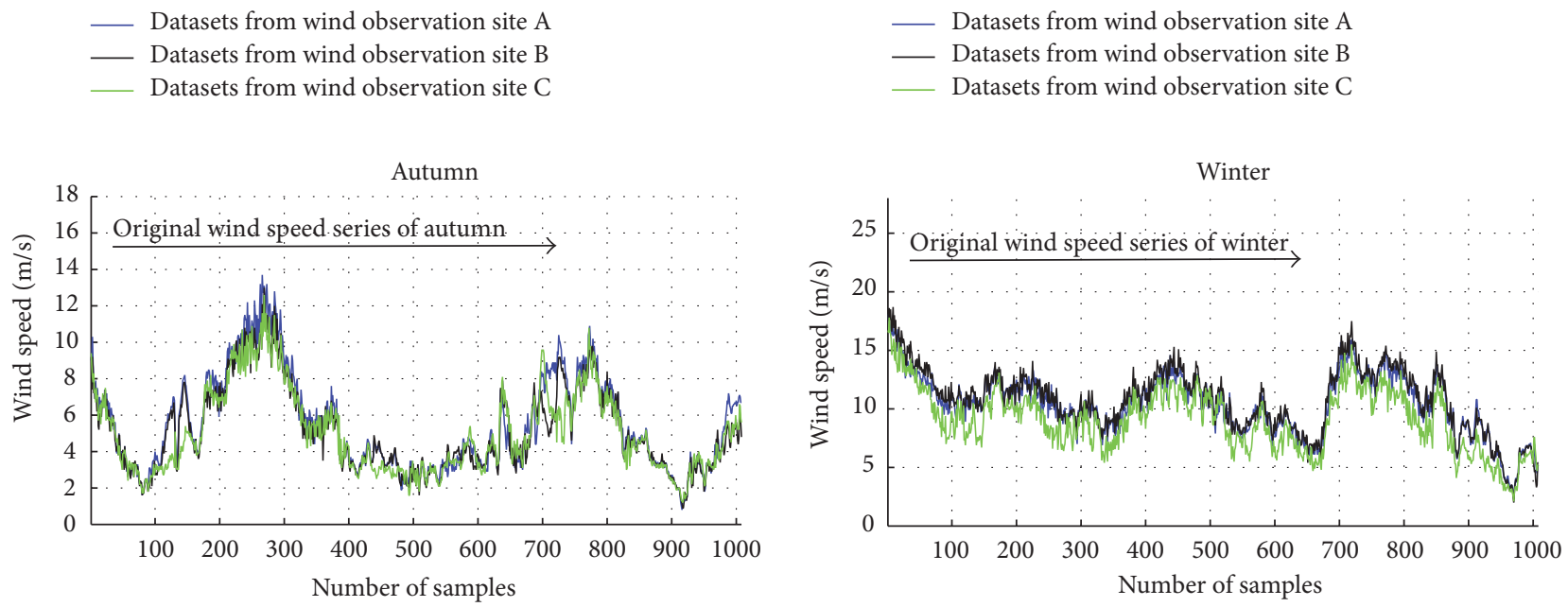

Datasets from wind observation site A
D Datasets from wind observation site B
Datasets from wind observation site C

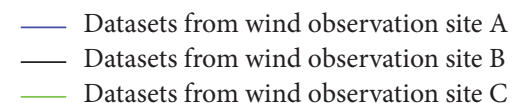

FIgURE 2: Four wind speed datasets (10 min speed) from three wind observation sites corresponding to the four seasons.

setting of SSA is very important for the forecasting effect. The window length $L$ is the only parameter in the SSA decomposition process. The window length was chosen as an integer fulfilling the conditions: $1<L<N$ and $L \leq N / 2$, where $N$ is the data length. In this paper, the data length of $10 \mathrm{~min}$ and $60 \mathrm{~min}$ wind speed is 1152 and 1032, respectively; therefore, the window length $L$ chosen is 400 .

Choosing $L=400$ allows the trend to be extracted simultaneously. Since the trend of wind speed series is complex, many eigentriples are required to reconstruct it. In this paper, the trend is reconstructed by eigentriples 1-150 in the 10 min wind speed forecasting experiments, and the $60 \mathrm{~min}$ wind speed trend is reconstructed by eigentriples 1100. The graph in Figure 4 depicts the initial series and trend of the $10 \mathrm{~min}$ wind speed series from the wind observation site $\mathrm{A}$.

3.3.2. Parameters of the Hybrid Model. Setting the parameters is very important for the prediction of wind speed. To compare the prediction effect of the model and attain a scientific conclusion, the initial parameters of these models need to be unified. The details are shown in Table 4 . 

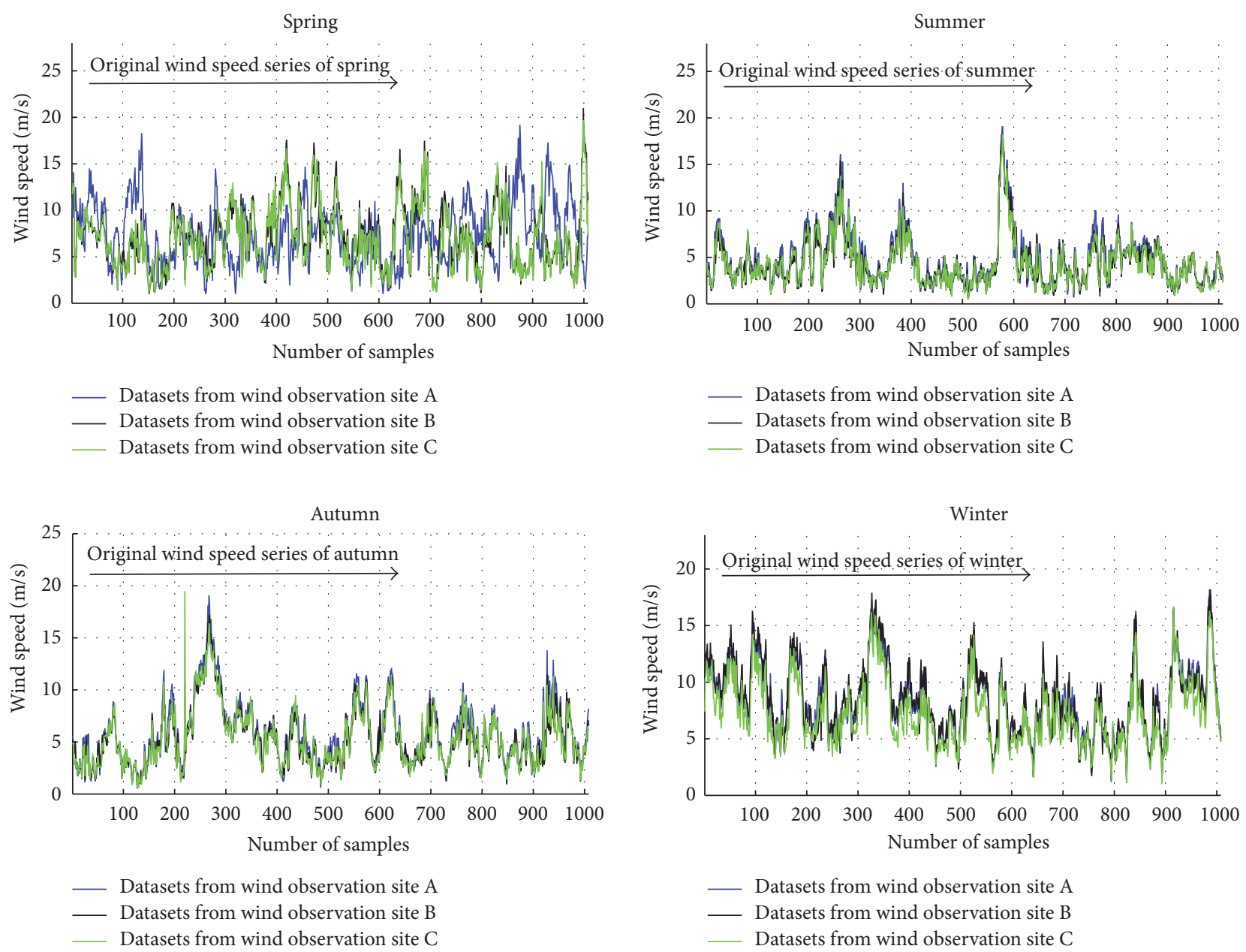

FIGURE 3: Four wind speed datasets (60 min speed) from three wind observation sites corresponding to the four seasons.

TABle 4: Parameters of the hybrid model.

\begin{tabular}{lcc}
\hline Experimental parameters & Default value \\
\hline & PSO & 1.494 \\
Acceleration coefficient & 1 \\
Inertia weight & 1000 \\
Maximum number of iterations & 20 \\
$\begin{array}{l}\text { The number of particles } \\
\text { Particle velocity }\end{array}$ & {$[-0.5,0.5]$} \\
Particle positions & {$[-5,5]$} \\
\hline & FA & 20 \\
The population size & & 50 \\
Maximum iteration number & & 1 \\
Absorption coefficient & & 0.2 \\
Light intensity coefficient & & 0.5 \\
Step size & & \\
Firefly positions & ENN & 4 \\
\hline & & 5 \\
Input layer & 1 \\
Middle layer & 1000 \\
Output layer & 0.000001 \\
Iteration time & 0.1 \\
Training requirement accuracy & \\
Learning rate &
\end{tabular}

3.4. Case Study One: 10 Min Wind Speed Forecasting. In this section, $10 \mathrm{~min}$ wind speed series, which are from four datasets of three wind observation sites, are used to test the forecasting capacity of the proposed hybrid model.

The forecasting results of the SSA-FAPSO-RENN model are compared with the forecasting results of the BPNN, ARIMA, RENN, SSA-RENN, and SSA-PSO-RENN. The BPNN, ARIMA, and RENN models are the single models, and the others are combination models. The parameters of BPNN are the same as those of ENN. The MAE, MAPE, and MSE values are the evaluation standard.

Figure 5 shows the multistep predicted results, the original wind speed data from spring datasets of wind observation site A, using the different involved models. The forecasting results are given in Tables 5-7. The values in bold indicate the smallest values of MSE, MAPE, and MAE. The smallest values of MSE, MAPE, and MAE are not all the forecasting results of the proposed model, but the minimum and the predictive values of the proposed model are very close. The results may differ with different error criterions. The proposed model is shown to have better prediction accuracy for most of the sample wind speed series. 


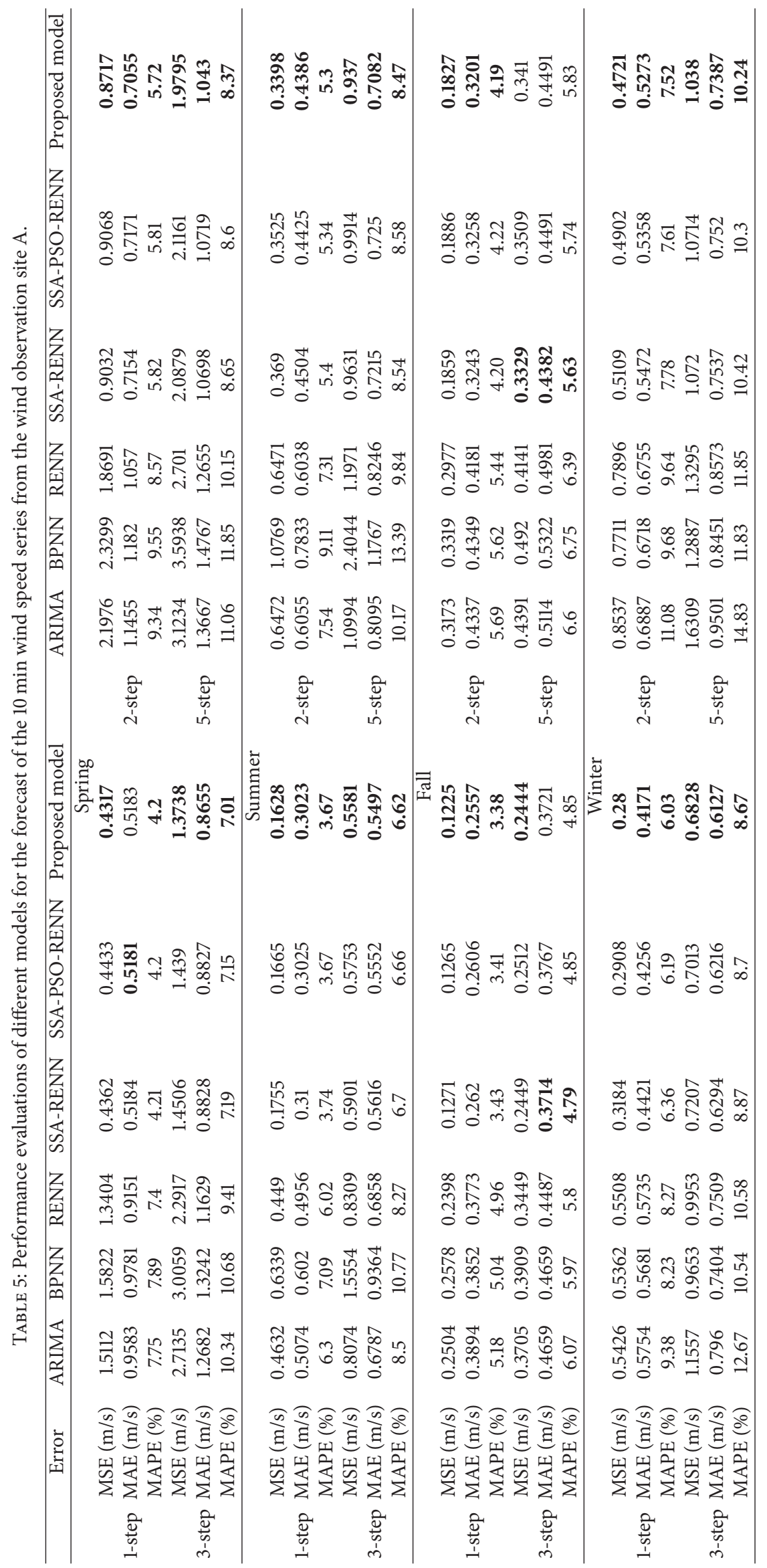




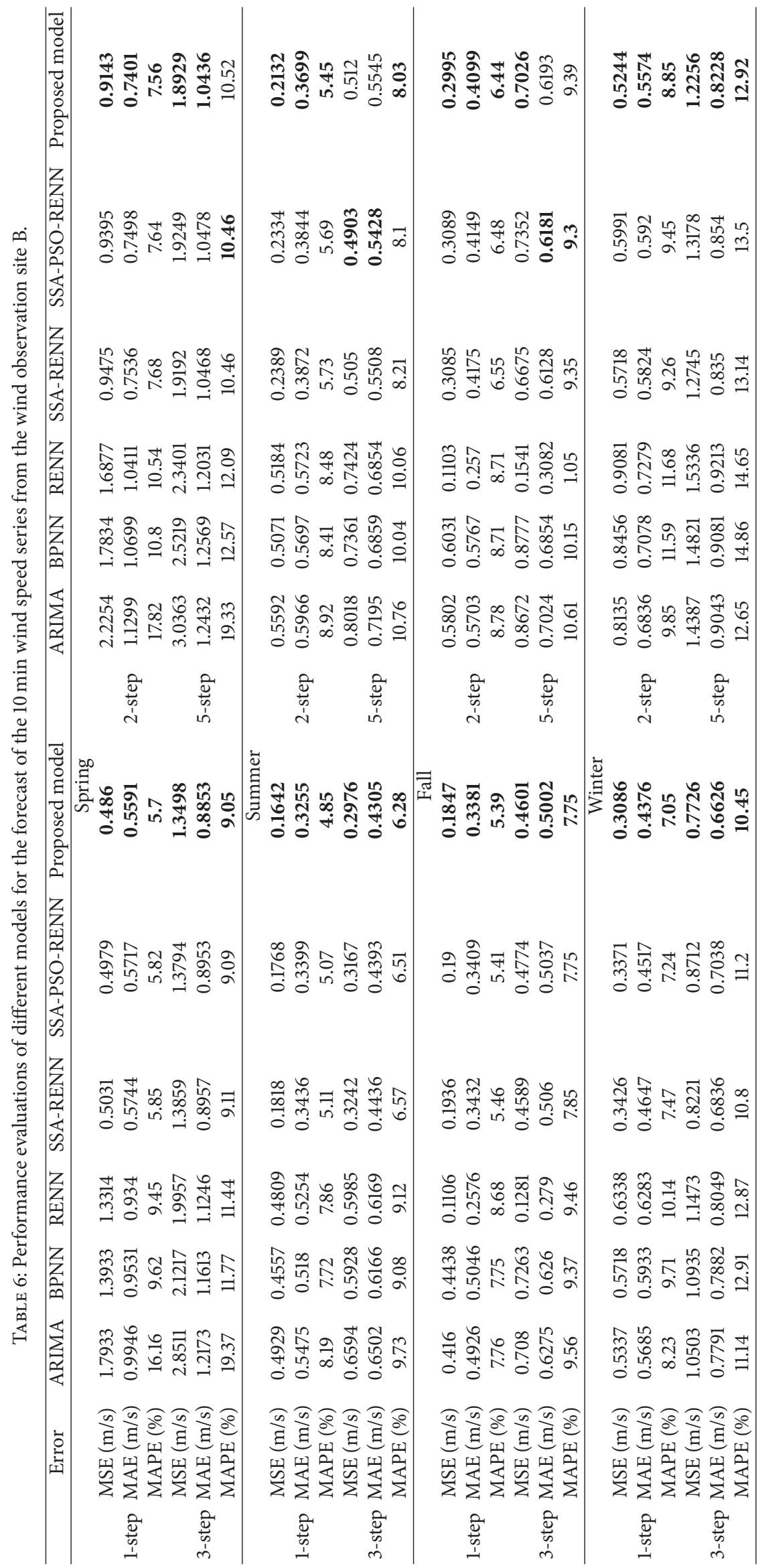




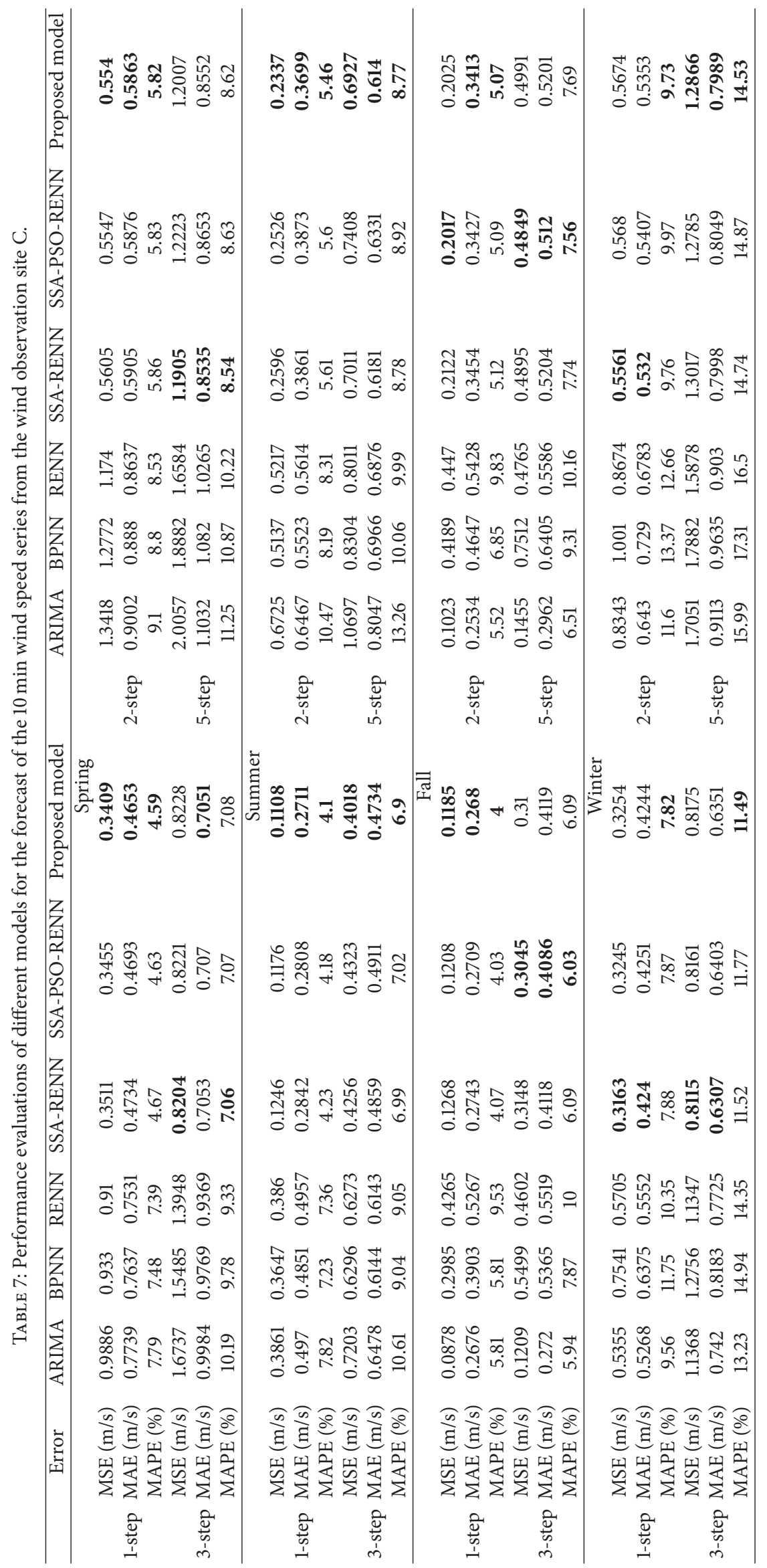



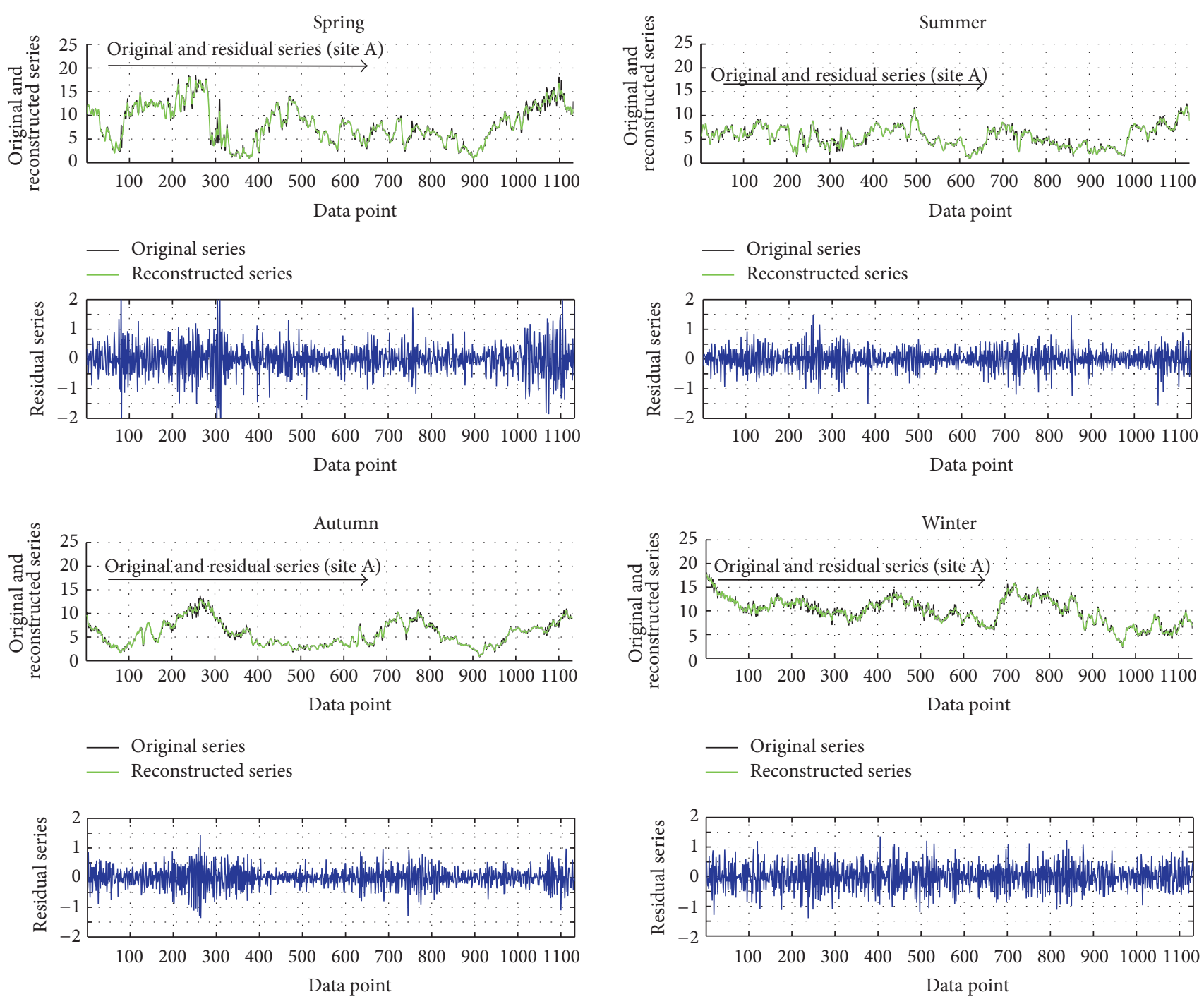

FIgURE 4: The initial series and trend of $10 \mathrm{~min}$ wind speed series from the wind observation site A.

To reflect the forecasting results more directly, the results of Tables 5-7 can be averaged. The average results were calculated by the results of three wind observation sites and four seasons. They are shown in Table 8.

Table 8 indicates the following:

(1) The forecasting results of 1-step are better than 2-step, 3 -step, and 5-step. For example, the MAPE values of the SSA-FAPSO-RENN model change from $5.06 \%$ to $6.43 \%, 7.69 \%$, and $9.45 \%$ at 2 -step, 3 -step, and 5-step. This conclusion can be reached through other models.

(2) Among all involved single models, the RENN model has the best performance except for the 1-step forecasting result, and the ARIMA model has the worst performance in every forecasting step.

(3) Compared with combined models, the single model forecasting effect is relatively poor.
(4) SSA-FAPSO-RENN offered the most accurate forecast value, with MAPE values of 5.06\%, 6.43\%, 7.69\%, and $9.45 \%$ at 1-step, 2 -step, 3 -step, and 5-step, respectively.

(5) The above conclusion can also be reached with MSE and MAE.

(6) The SSA can improve the forecasting performance of the RENN model.

Wind speed high-accuracy forecasting, an important part of electrical system monitoring and control, is crucial to protect the safety of wind power utilization but is always a difficult and arduous task. Compared with the other forecasting models involved in this paper, the proposed hybrid model has better forecasting ability in the $10 \mathrm{~min}$ wind speed forecasting study.

3.5. Case Study Two: 60 Min Wind Speed Forecasting. In this case, one-hour wind speed series were used to test the 

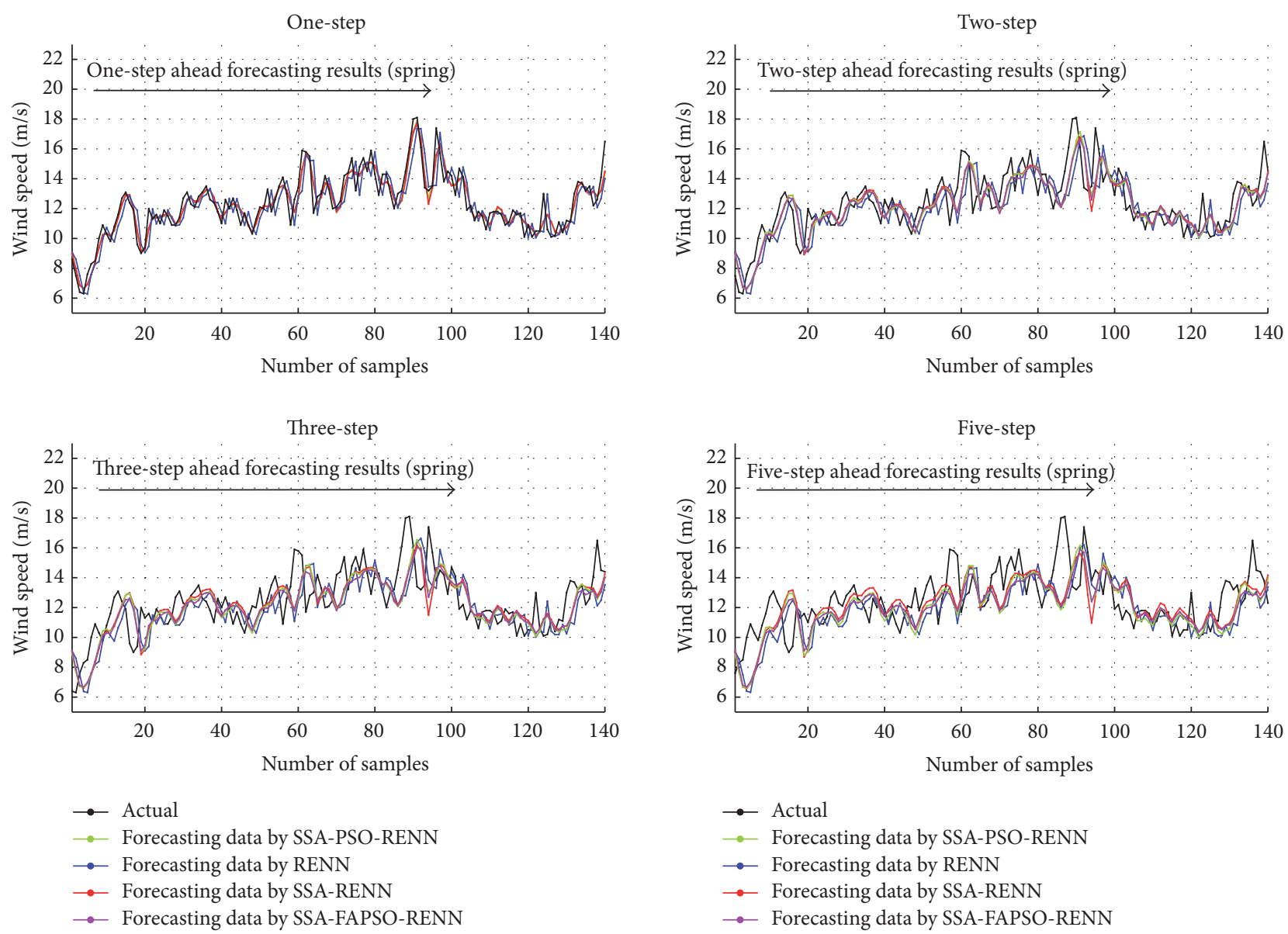

$\rightarrow$ Actual
$\because$ Forecasting data by SSA-PSO-RENN
$\rightarrow-$ Forecasting data by RENN
$\rightarrow-$ Forecasting data by SSA-RENN
$\rightarrow$ Forecasting data by SSA-FAPSO-RENN

FIGURE 5: The multistep predicted results of $10 \mathrm{~min}$ wind speed series using the different involved models.

TABLE 8: Average results of the $10 \mathrm{~min}$ wind speed.

\begin{tabular}{lcccc}
\hline & 1-step & \multicolumn{4}{c}{ 2-step } & 3-step & 5-step \\
\hline \multirow{4}{*}{ MSE (m/s) } & 0.6668 & 0.9287 & 1.164 & 1.4469 \\
MAE (m/s) & 0.5916 & 0.6914 & 0.7619 & 0.8602 \\
MAPE (\%) & 8.33 & 9.64 & 10.61 & 11.92 \\
\hline \multicolumn{5}{c}{ RENN } \\
MSE (m/s) & 0.6191 & 0.8198 & 0.9958 & 1.2446 \\
MAE (m/s) & 0.5865 & 0.6666 & 0.7291 & 0.8116 \\
MAPE (\%) & 8.12 & 9.14 & 9.97 & 10.25 \\
\hline & \multicolumn{5}{c}{ SSA-PSO-RENN } \\
MSE (m/s) & 0.2614 & 0.4663 & 0.6989 & 1.0604 \\
MAE (m/s) & 0.388 & 0.5017 & 0.6021 & 0.7397 \\
MAPE (\%) & 5.1436 & 6.5611 & 7.8166 & 9.5465 \\
\hline & \multicolumn{5}{c}{ BPNN } \\
MSE (m/s) & 0.6854 & 0.955 & 1.2046 & 1.5546 \\
MAE (m/s) & 0.6149 & 0.7192 & 0.8004 & 0.9125 \\
MAPE (\%) & 7.94 & 9.22 & 10.23 & 11.58 \\
\hline & \multicolumn{5}{c}{ SSA-RENN } \\
MSE (m/s) & 0.2664 & 0.4687 & 0.6975 & 1.0421 \\
MAE (m/s) & 0.3929 & 0.5027 & 0.6006 & 0.735 \\
MAPE (\%) & 5.21 & 6.57 & 7.8 & 9.52 \\
\hline & \multicolumn{5}{c}{ SSA-FAPSO-RENN } \\
MSE (m/s) & $\mathbf{0 . 2 5 3}$ & $\mathbf{0 . 4 4 7 9}$ & $\mathbf{0 . 6 7 4 3}$ & $\mathbf{1 . 0 2 5 6}$ \\
MAE (m/s) & $\mathbf{0 . 3 8 1 9}$ & $\mathbf{0 . 4 9 1 8}$ & $\mathbf{0 . 5 9 2}$ & $\mathbf{0 . 7 3 0 6}$ \\
MAPE (\%) & $\mathbf{5 . 0 6 5 7}$ & $\mathbf{6 . 4 2 6 4}$ & $\mathbf{7 . 6 8 6 9}$ & $\mathbf{9 . 4 4 8 7}$ \\
\hline
\end{tabular}

forecasting capacity of the proposed hybrid model. Figure 6 depicts the initial series and trend of the $60 \mathrm{~min}$ wind speed series from the wind observation site A.

The forecasting results of proposed hybrid model, SSAFAPSO-RENN, are compared with the forecasting results of BPNN, ARIMA, RENN, SSA-RENN, and SSA-PSO-RENN.

Figure 7 shows the multistep predicted results with the one-hour wind speed data from spring datasets of wind observation site A, using the different involved models. The estimated results of these predictions are given in Tables 9-11.

From Tables 9-11, the values in bold indicate the smallest values of MSE, MAPE, and MAE, and the minimum values and the predictive value of the proposed model are approximately the same; compared to $10 \mathrm{~min}$ forecasting results, onehour wind speed forecasting has more error; with the increase of prediction steps, precision will rise; some wind speed series are more adaptable to other models, but overall, the proposed model has better prediction ability.

To reflect the forecasting results more directly, the results of Tables 9-11 can be averaged. The average results are shown in Table 12.

Table 12 indicates the following:

(7) The forecasting results of 1-step are better than 2-step, 3-step, and 5-step. The MAPE values of the proposed 

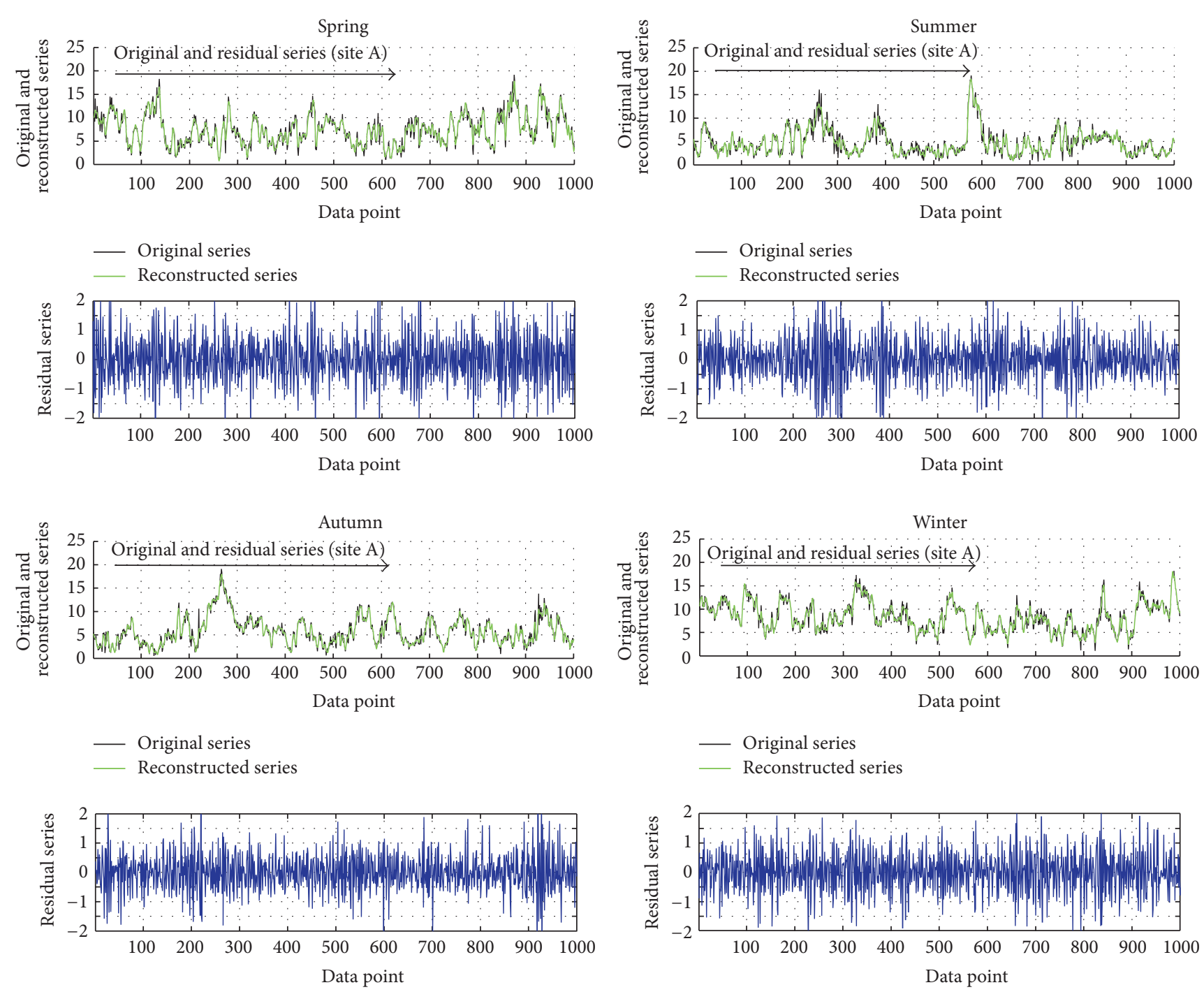

FIGURE 6: The initial series and trend of the $60 \mathrm{~min}$ wind speed series from wind observation site A.

model change from $10.23 \%$ to $11.78 \%, 13.39 \%$, and $15.50 \%$ at 2 -step, 3-step, and 5-step. Other models can also reach this conclusion.

(8) Among all involved single models, the RENN model has the best performance in every forecasting step, and the ARIMA model has the worst performance. The MAPE values of the ARIMA model are $15.65 \%$, $17.61 \%, 19.03 \%$, and $21.02 \%$ at 1-step, 2-step, 3-step, and 5-step, respectively. However, the values of RENN reduce to $12.92 \%, 14.40 \%, 15.36 \%$, and $16.67 \%$.

(9) SSA-FAPSO-RENN offered the most accurate forecast value. In 1-step forecasting experiment, the MAPE values of the combined model were reduced by $20.8 \%, 3.8 \%$, and $2.1 \%$ compared with the Elman model, SSA Elman, and SSAFA Elman, respectively. Similar conclusions can be obtained from multistep experiments.
(10) The above conclusion can also be achieved with MSE and MAE.

The forecasting results are generally good. The proposed hybrid model can be used to forecast $60 \mathrm{~min}$ wind speed. Compared with traditional single models and other models involved in this paper, the proposed model has the best forecasting ability. The forecasting results show that the model has a better performance in the $10 \mathrm{~min}$ wind speed forecasting study than the 60 min study.

The average results of study one and study two are shown in Table 13. The proposed model has the best performance as evaluated by MAPE, MAE, and MSE. The Diebold-Mariano values of the SSA-PSO-RENN and SSA-RENN models are larger than the upper limits at the $10 \%$ significance level, the DM values of the RENN and the BP models are larger than the upper limits at the 5\% significance level, and the DM value of the ARIMA models is larger than the upper limits at the 


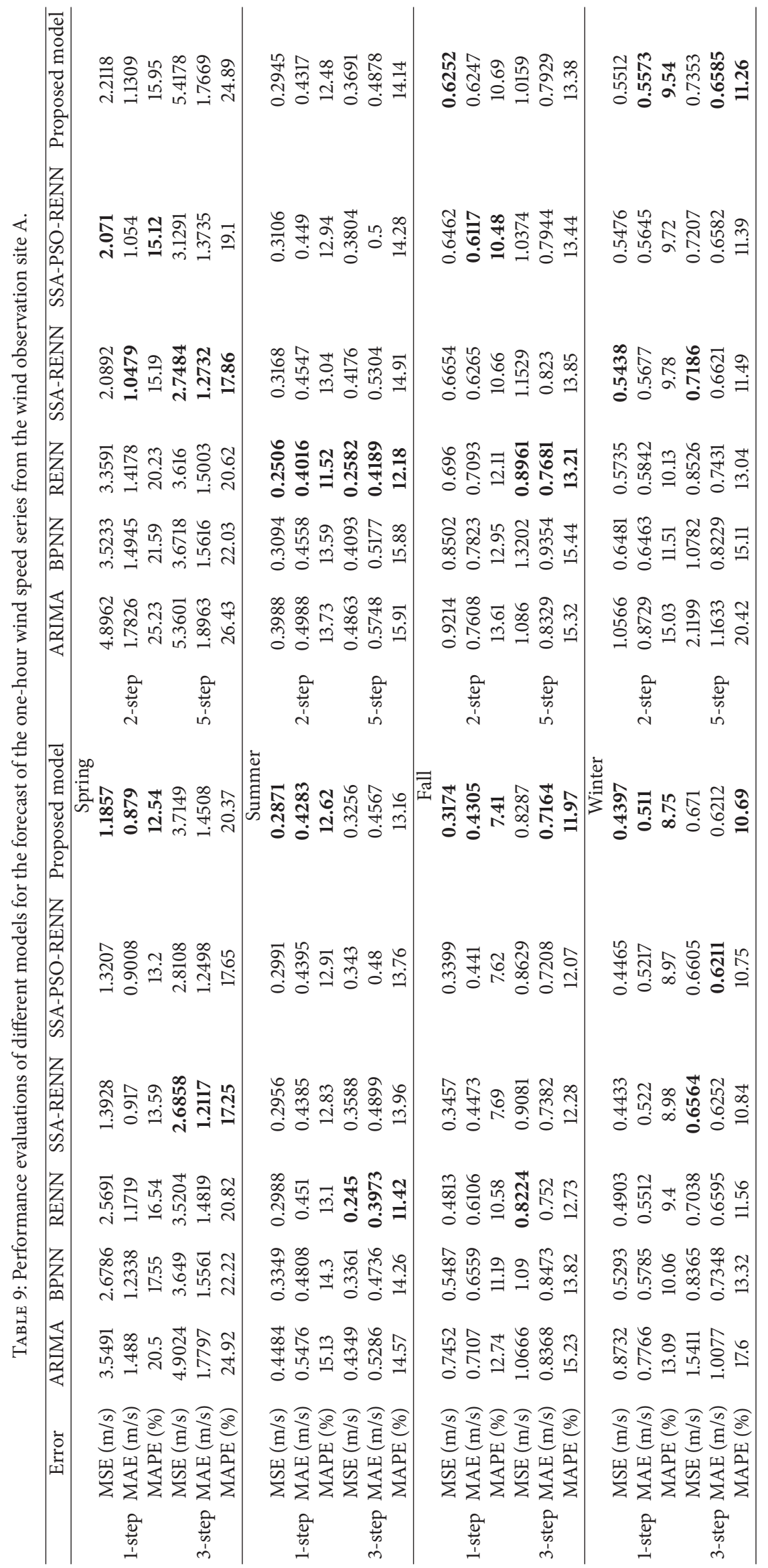




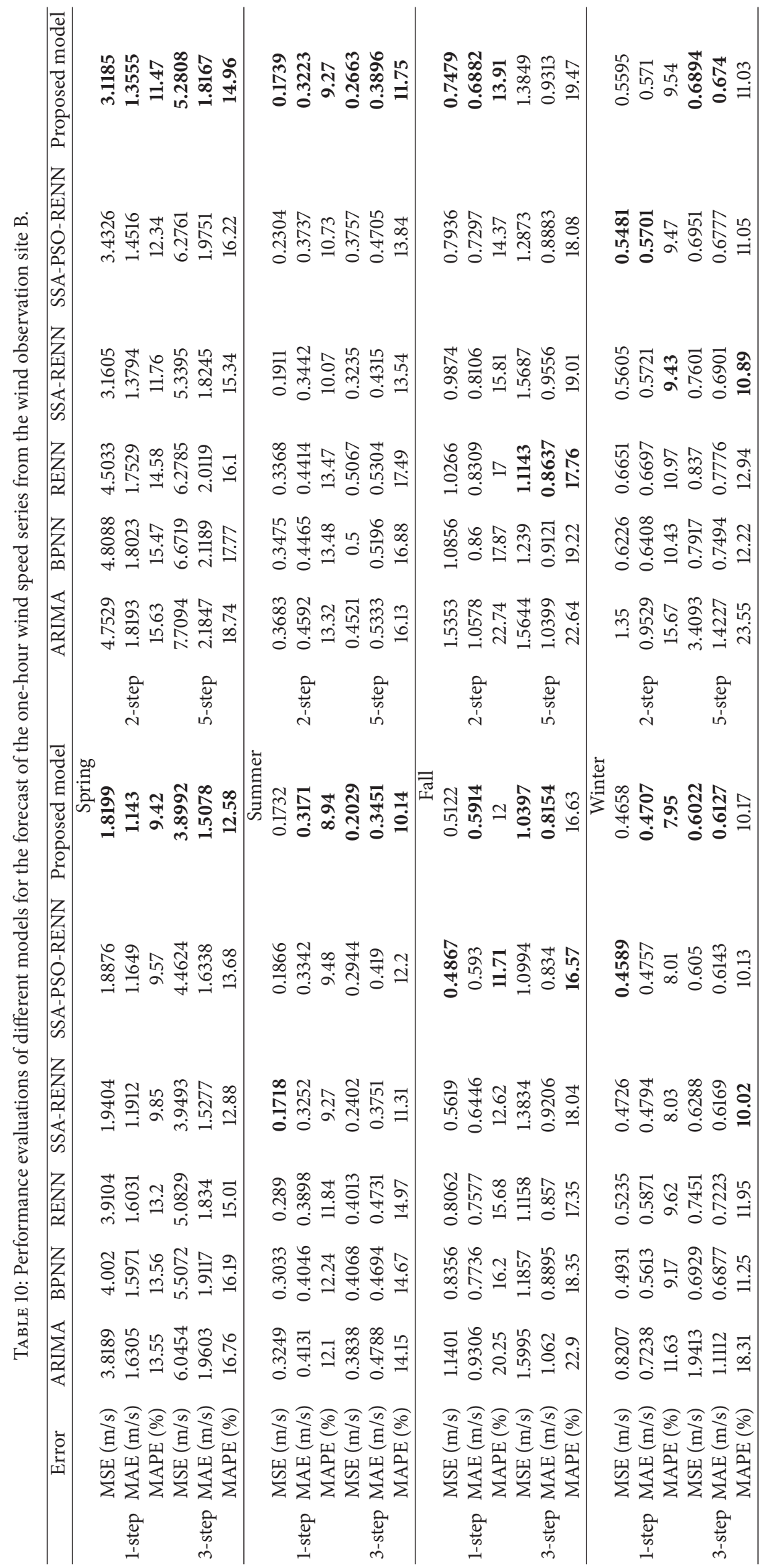




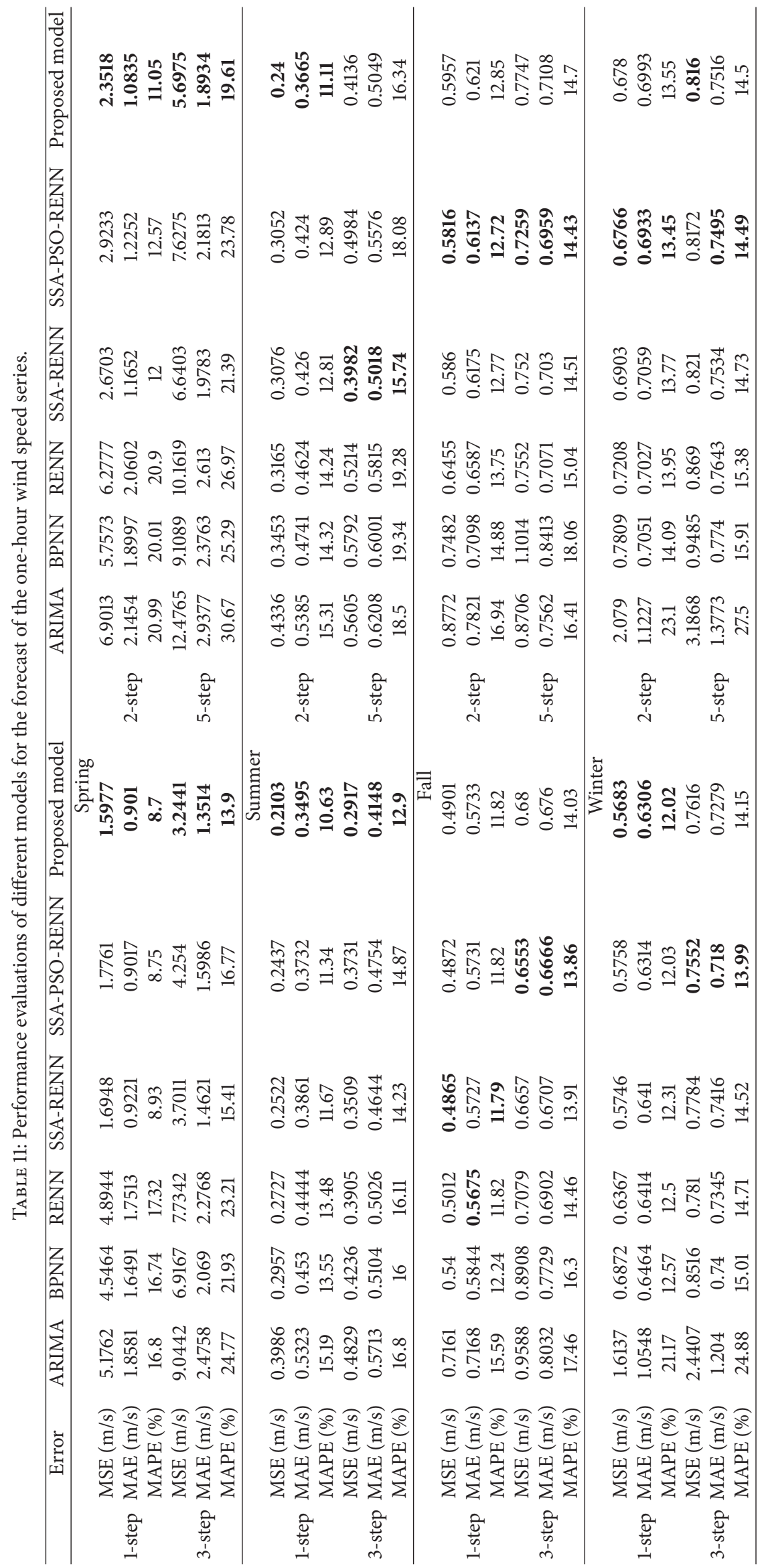



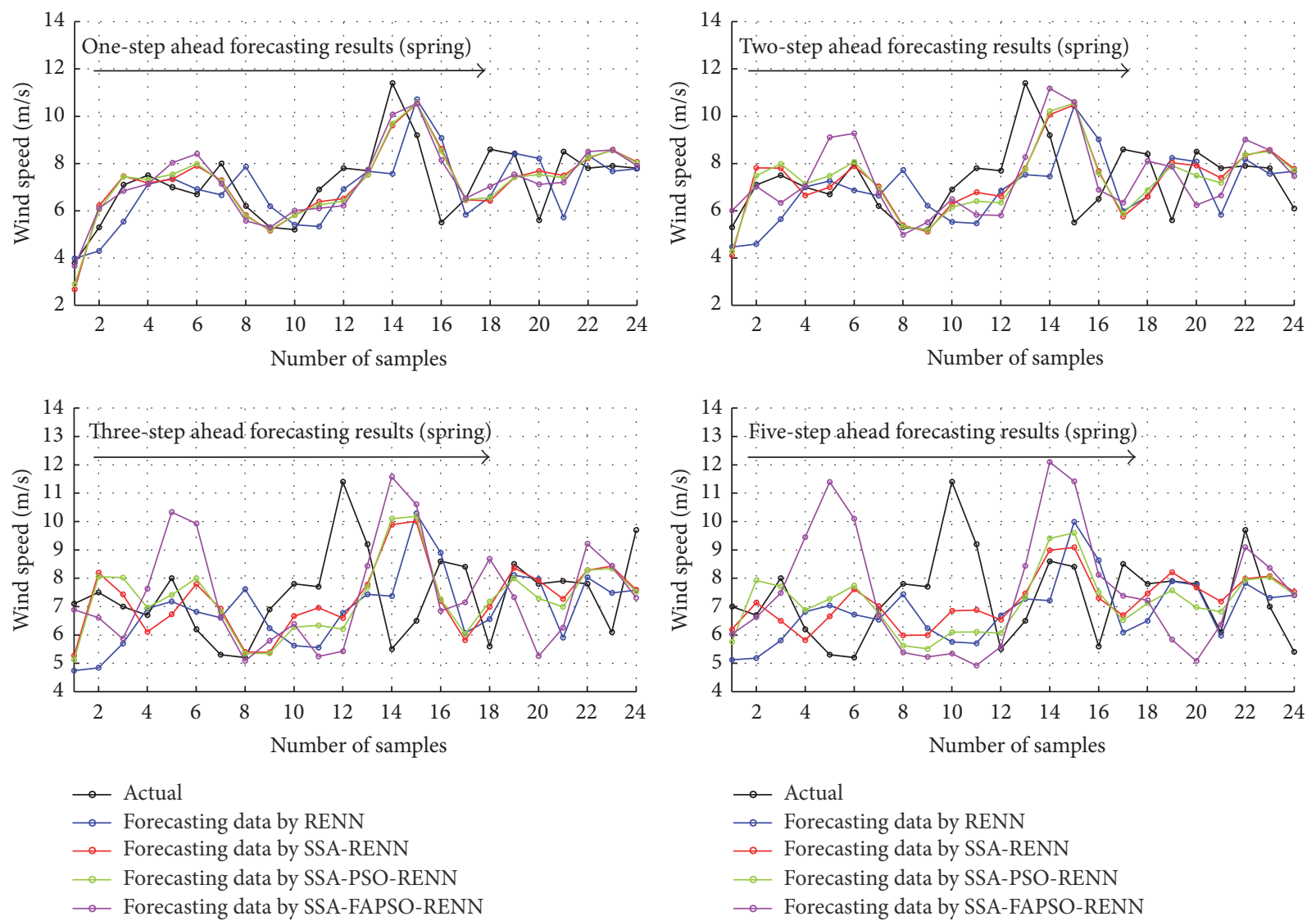

FIGURE 7: The multistep predicted results of one-hour wind speed series using the different involved models.

TABLE 12: Average results of one-hour wind speed.

\begin{tabular}{|c|c|c|c|c|}
\hline & 1-step & 2-step & 3-step & 5-step \\
\hline & \multicolumn{4}{|c|}{ ARIMA } \\
\hline $\operatorname{MSE}(\mathrm{m} / \mathrm{s})$ & 1.6354 & 2.1309 & 2.5701 & 3.2735 \\
\hline $\operatorname{MAE}(\mathrm{m} / \mathrm{s})$ & 0.9486 & 1.0661 & 1.1516 & 1.2783 \\
\hline \multirow[t]{2}{*}{ MAPE (\%) } & 15.65 & 17.61 & 19.03 & 21.02 \\
\hline & \multicolumn{4}{|c|}{ RENN } \\
\hline $\operatorname{MSE}(\mathrm{m} / \mathrm{s})$ & 1.2826 & 1.644 & 1.9423 & 2.359 \\
\hline $\operatorname{MAE}(\mathrm{m} / \mathrm{s})$ & 0.7939 & 0.891 & 0.9484 & 1.0233 \\
\hline \multirow[t]{2}{*}{ MAPE (\%) } & 12.92 & 14.4 & 15.36 & 16.67 \\
\hline & \multicolumn{4}{|c|}{ SSA-PSO-RENN } \\
\hline $\operatorname{MSE}(\mathrm{m} / \mathrm{s})$ & 0.71 & 1.0889 & 1.4313 & 1.9642 \\
\hline $\operatorname{MAE}(\mathrm{m} / \mathrm{s})$ & 0.6125 & 0.73 & 0.8369 & 0.9602 \\
\hline \multirow[t]{2}{*}{ MAPE (\%) } & 10.45 & 12.23 & 13.86 & 15.68 \\
\hline & \multicolumn{4}{|c|}{ BPNN } \\
\hline $\operatorname{MSE}(\mathrm{m} / \mathrm{s})$ & 1.3162 & 1.6523 & 1.8989 & 2.285 \\
\hline $\operatorname{MAE}(\mathrm{m} / \mathrm{s})$ & 0.8015 & 0.9098 & 0.9719 & 1.0608 \\
\hline \multirow[t]{2}{*}{ MAPE (\%) } & 13.28 & 15.02 & 16.11 & 17.76 \\
\hline & \multicolumn{4}{|c|}{ SSA-RENN } \\
\hline $\operatorname{MSE}(\mathrm{m} / \mathrm{s})$ & 0.72 & 1.064 & 1.3589 & 1.8034 \\
\hline $\operatorname{MAE}(\mathrm{m} / \mathrm{s})$ & 0.6239 & 0.7265 & 0.8203 & 0.9272 \\
\hline \multirow[t]{2}{*}{ MAPE (\%) } & 10.63 & 12.26 & 13.72 & 15.27 \\
\hline & \multicolumn{4}{|c|}{ SSA-FAPSO-RENN } \\
\hline $\operatorname{MSE}(\mathrm{m} / \mathrm{s})$ & 0.6723 & 1.0123 & 1.3551 & 1.9051 \\
\hline $\operatorname{MAE}(\mathrm{m} / \mathrm{s})$ & 0.6021 & 0.7043 & 0.808 & 0.9482 \\
\hline MAPE (\%) & 10.23 & 11.78 & 13.39 & 15.5 \\
\hline
\end{tabular}

$1 \%$ significance level. The result indicates that the proposed hybrid model is significantly better than other models.

\section{Conclusions}

Wind power systems need to further develop accurate and reliable technology for short-term wind speed forecasting. Due to the influence of various meteorological factors, wind speed series are intermittent and randomly characterized, making it difficult to forecast wind speed using a single model. The focus of recent research has been the development of new methods and combinations of methods. However, individual models do not always achieve desirable performance. Hybrid models can decrease negative influences that are intrinsic in each of the individual models. These models can use the advantages of each individual model and are less sensitive, in certain cases, to the factors that cause the individual models to perform undesirably. Therefore, the hybrid model is more effective than individual models for wind speed forecasting.

To forecast the $10 \mathrm{~min}$ and one-hour wind speed more accurately, a new hybrid model, SSA-FAPSO-RENN, is proposed, which can overcome many limitations of single models, such as poor prediction accuracy and artificial parameters. The forecasting results show that the proposed model can improve the accuracy of $10 \mathrm{~min}$ and $60 \mathrm{~min}$ wind speed forecasting. Compared with other models involved in 
TABLE 13: The average results of study one and study two.

\begin{tabular}{lcccccc}
\hline & ARIMA & BP & RENN & SSA-RENN & SSA-PSO-RENN & Proposed model \\
\hline MSE (m/s) & 1.7270 & 1.4440 & 1.3634 & 0.9276 & 0.9602 & 0.6714 \\
MAE (m/s) & 0.9187 & 0.8489 & 0.8063 & 0.6661 & 10.12 & 10.16 \\
MAPE (\%) & 14.23 & 12.64 & 12.10 & $1.89^{* * *}$ & $1.74^{* * *}$ & $\mathbf{0 . 6 5 7 4}$ \\
DM & $2.57^{* *}$ & $2.67^{*}$ & $2.45^{* *}$ & $\mathbf{9 . 9 4}$ & - \\
\hline
\end{tabular}

$*$ is $1 \%$ significance level; $* *$ is $5 \%$ significance level; $* * *$ is $10 \%$ significance level.

this paper, the prediction precision of the proposed model is the largest.

\section{Competing Interests}

The authors declare that they have no competing interests.

\section{Acknowledgments}

This work was supported by the National Social Science Foundation of China (Grant no. 13\&ZD171).

\section{References}

[1] H. Liu, H.-Q. Tian, C. Chen, and Y.-F. Li, "A hybrid statistical method to predict wind speed and wind power," Renewable Energy, vol. 35, no. 8, pp. 1857-1861, 2010.

[2] M. Yesilbudak, S. Sagiroglu, and I. Colak, "A new approach to very short term wind speed prediction using k-nearest neighbor classification," Energy Conversion and Management, vol. 69, pp. 77-86, 2013.

[3] H. Holttinen, "Optimal electricity market for wind power," Energy Policy, vol. 33, no. 16, pp. 2052-2063, 2005.

[4] J. Wang, S. Qin, Q. Zhou, and H. Jiang, "Medium-term wind speeds forecasting utilizing hybrid models for three different sites in Xinjiang, China," Renewable Energy, vol. 76, pp. 91-101, 2015.

[5] M. Lei, L. Shiyan, J. Chuanwen, L. Hongling, and Z. Yan, "A review on the forecasting of wind speed and generated power," Renewable and Sustainable Energy Reviews, vol. 13, no. 4, pp. 915-920, 2009.

[6] F. Cassola and M. Burlando, "Wind speed and wind energy forecast through Kalman filtering of Numerical Weather Prediction model output," Applied Energy, vol. 99, pp. 154-166, 2012.

[7] L. Landberg, "Short-term prediction of local wind conditions," Journal of Wind Engineering and Industrial Aerodynamics, vol. 89, no. 3-4, pp. 235-245, 2001.

[8] M. S. Miranda and R. W. Dunn, "One-hour-ahead wind speed prediction using a Bayesian methodology," in Proceedings of the IEEE Power Engineering Society General Meeting (PES '06), June 2006.

[9] G. H. Riahy and M. Abedi, "Short term wind speed forecasting for wind turbine applications using linear prediction method," Renewable Energy, vol. 33, no. 1, pp. 35-41, 2008.

[10] H. Liu, J. Shi, and E. Erdem, "Prediction of wind speed time series using modified Taylor Kriging method," Energy, vol. 35, no. 12 , pp. $4870-4879,2010$.
[11] H. Kantz, D. Holstein, M. Ragwitz, and N. K. Vitanov, "Markov chain model for turbulent wind speed data," Physica A: Statistical Mechanics and Its Applications, vol. 342, no. 1-2, pp. 315-321, 2004.

[12] E. Erdem and J. Shi, "ARMA based approaches for forecasting the tuple of wind speed and direction," Applied Energy, vol. 88, no. 4, pp. 1405-1414, 2011.

[13] J. L. Torres, A. García, M. De Blas, and A. De Francisco, "Forecast of hourly average wind speed with ARMA models in Navarre (Spain)," Solar Energy, vol. 79, no. 1, pp. 65-77, 2005.

[14] P. Areekul, T. Senjyu, H. Toyama, and A. Yona, "A hybrid ARIMA and neural network model for short-term price forecasting in deregulated market," IEEE Transactions on Power Systems, vol. 25, no. 1, pp. 524-530, 2010.

[15] P. Chen, T. Pedersen, B. Bak-Jensen, and Z. Chen, "ARIMAbased time series model of stochastic wind power generation," IEEE Transactions on Power Systems, vol. 25, no. 2, pp. 667-676, 2010.

[16] H. Liu, H.-Q. Tian, D.-F. Pan, and Y.-F. Li, "Forecasting models for wind speed using wavelet, wavelet packet, time series and Artificial Neural Networks," Applied Energy, vol. 107, pp. 191208, 2013.

[17] L. Xiao, J. Wang, R. Hou, and J. Wu, "A combined model based on data pre-analysis and weight coefficients optimization for electrical load forecasting," Energy, vol. 82, pp. 524-549, 2015.

[18] T. G. Barbounis, J. B. Theocharis, M. C. Alexiadis, and P. S. Dokopoulos, "Long-term wind speed and power forecasting using local recurrent neural network models," IEEE Transactions on Energy Conversion, vol. 21, no. 1, pp. 273-284, 2006.

[19] Y.-Y. Hong, H.-L. Chang, and C.-S. Chiu, "Hour-ahead wind power and speed forecasting using simultaneous perturbation stochastic approximation (SPSA) algorithm and neural network with fuzzy inputs," Energy, vol. 35, no. 9, pp. 3870-3876, 2010.

[20] G. Sideratos and N. D. Hatziargyriou, "An advanced statistical method for wind power forecasting," IEEE Transactions on Power Systems, vol. 22, no. 1, pp. 258-265, 2007.

[21] S. A. Pourmousavi Kani and G. H. Riahy, "A new ANN-based methodology for very short-term wind speed prediction using Markov chain approach," in Proceedings of the IEEE Electrical Power and Energy Conference, pp. 6-7, October 2008.

[22] T. H. M. El-Fouly, E. F. El-Saadany, and M. M. A. Salama, "One day ahead prediction of wind speed using annual trends," in Proceedings of the IEEE Power Engineering Society General Meeting (PES '06), June 2006.

[23] E. Cadenas and W. Rivera, "Wind speed forecasting in the South Coast of Oaxaca, México," Renewable Energy, vol. 32, no. 12, pp. 2116-2128, 2007.

[24] X. C. Wang, P. Guo, and X. B. Huang, "A review of wind power forecasting models," Energy Procedia, vol. 12, pp. 770-778, 2011. 
[25] M. A. Mohandes, T. O. Halawani, S. Rehman, and A. A. Hussain, "Support vector machines for wind speed prediction," Renewable Energy, vol. 29, no. 6, pp. 939-947, 2004.

[26] M. Carolin Mabel and E. Fernandez, "Analysis of wind power generation and prediction using ANN: A case study," Renewable Energy, vol. 33, no. 5, pp. 986-992, 2008.

[27] J. Wang, W. Zhu, W. Zhang, and D. Sun, "A trend fixed on firstly and seasonal adjustment model combined with thee-SVR for short-term forecasting of electricity demand," Energy Policy, vol. 37, no. 11, pp. 4901-4909, 2009.

[28] F. Yu and X. Xu, "A short-term load forecasting model of natural gas based on optimized genetic algorithm and improved BP neural network," Applied Energy, vol. 134, pp. 102-113, 2014.

[29] G. Xu, C. Xiu, and Z. Wan, "Hysteretic chaotic operator network and its application in wind speed series prediction," Neurocomputing, vol. 165, pp. 384-388, 2015.

[30] B. H. Petkov, V. Vitale, M. Mazzola, C. Lanconelli, and A. Lupi, "Chaotic behaviour of the short-term variations in ozone column observed in Arctic," Communications in Nonlinear Science and Numerical Simulation, vol. 26, no. 1-3, pp. 238-249, 2015.

[31] L. Yang, J. Zhang, X. Wu, Y. Zhang, and J. Li, "A chaotic time series prediction model for speech signal encoding based on genetic programming," Applied Soft Computing Journal, vol. 38, pp. 754-761, 2016.

[32] X. An, D. Jiang, M. Zhao, and C. Liu, "Short-term prediction of wind power using EMD and chaotic theory," Communications in Nonlinear Science and Numerical Simulation, vol. 17, no. 2, pp. 1036-1042, 2012.

[33] J. P. S. Catalão, H. M. I. Pousinho, and V. M. F. Mendes, "Shortterm wind power forecasting in Portugal by neural networks and wavelet transform," Renewable Energy, vol. 36, no. 4, pp. 1245-1251, 2011.

[34] S. Huang, J. Chang, Q. Huang, and Y. Chen, "Monthly streamflow prediction using modified EMD-based support vector machine," Journal of Hydrology, vol. 511, pp. 764-775, 2014.

[35] P. Du, Y. Jin, and K. Zhang, "A hybrid multi-step rolling forecasting model based on ssa and simulated annealing-adaptive particle swarm optimization for wind speed," Sustainability, vol. 8, no. 8, article 754, 2016.

[36] A. Alvarez, "Performance of Satellite-based Ocean Forecasting (SOFT) systems: a study in the Adriatic Sea," Journal of Atmospheric and Oceanic Technology, vol. 20, no. 5, pp. 717-729, 2003.

[37] C. Penland, M. Ghil, and K. M. Weickmann, "Adaptive filtering and maximum entropy spectra with application to changes in atmospheric angular momentum," Journal of Geophysical Research, vol. 96, no. 22, pp. 659-671, 1991.

[38] M. Casdagli, D. Des Jardins, and S. Eubank, "Nonlinear modeling of chaotic time series: theory and applications," in Applied Chaos, J. H. Kim and J. Stringer, Eds., pp. 335-380, John Wiley \& Sons, 1992.

[39] R. Chandra, "Competition and collaboration in cooperative coevolution of Elman recurrent neural networks for timeseries prediction," IEEE Transactions on Neural Networks and Learning Systems, vol. 26, no. 12, pp. 3123-3136, 2015.

[40] X.-S. Yang, "Firefly algorithms for multimodal optimization," in Stochastic Algorithms: Foundations and Applications, vol. 5792 of Lecture Notes in Computer Science, pp. 169-178, Springer, Berlin, Germany, 2009.
[41] X.-S. Yang, "Chaos-enhanced firefly algorithm with automatic parameter tuning," The International Journal of Swarm Intelligence Research (IJSIR), vol. 2, pp. 1-11, 2011.

[42] J. Kennedy and R. Eberhart, "Particle swarm optimization," in Proceedings of the IEEE International Conference on Neural Networks, vol. 4, pp. 1942-1948, 1995.

[43] F. Pan, Q. Zhou, W. X. Li, and Q. Gao, "Analysis of the standard particle swarm optimization algorithm based on Markov chain," Acta Automatica Sinica, vol. 39, no. 4, pp. 381-389, 2013.

[44] F. X. Diebold and R. Mariano, "Comparing predictive accuracy," Journal of Business \& Economic Statistics, vol. 13, pp. 253-263, 1995. 


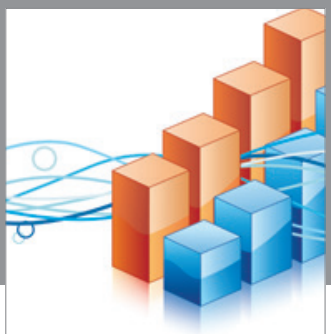

Advances in

Operations Research

vatem alat4

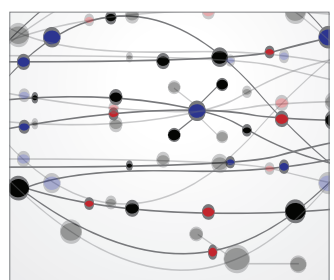

\section{The Scientific} World Journal
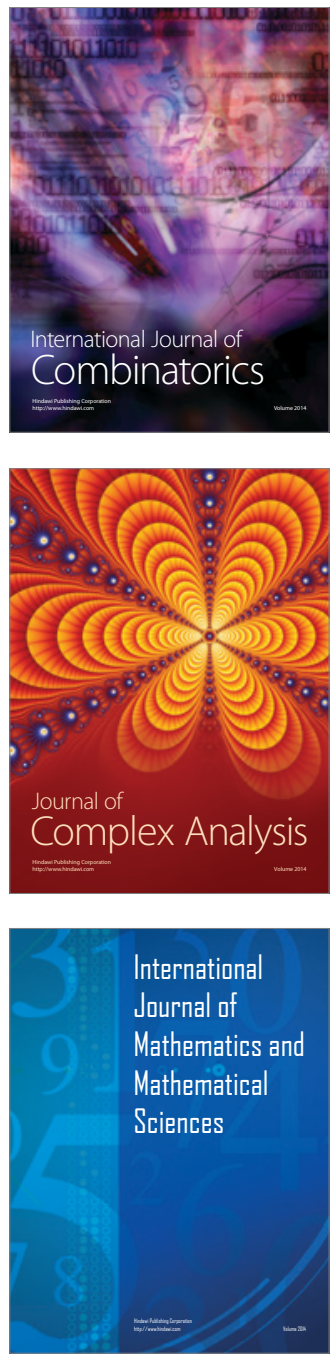
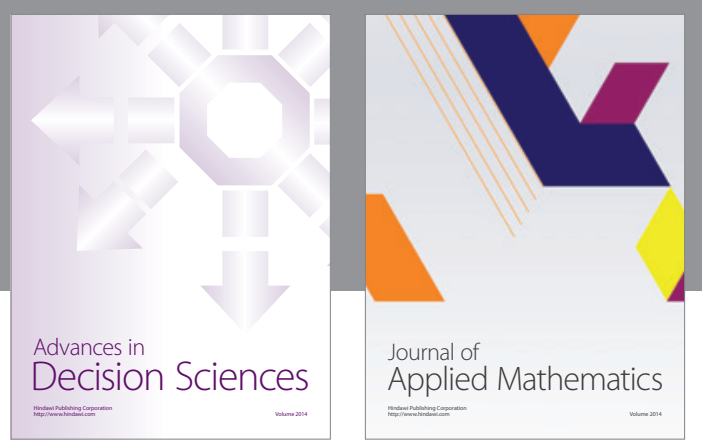

Algebra

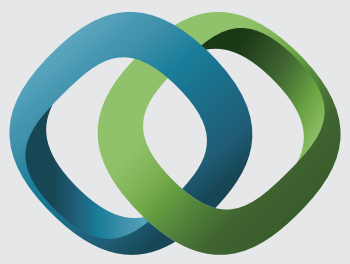

\section{Hindawi}

Submit your manuscripts at

http://www.hindawi.com
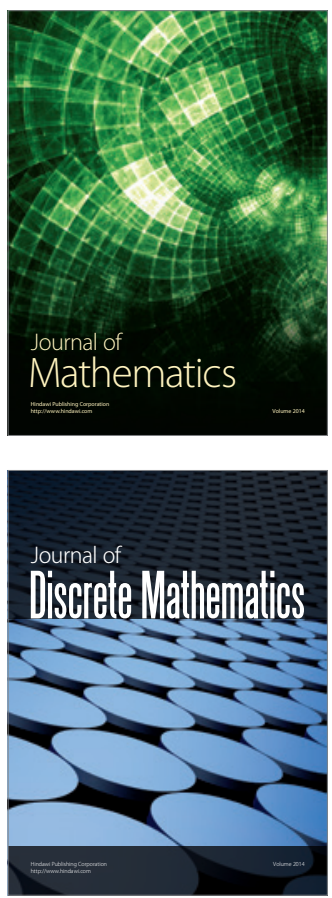

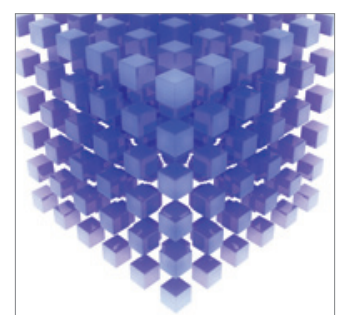

Mathematical Problems in Engineering
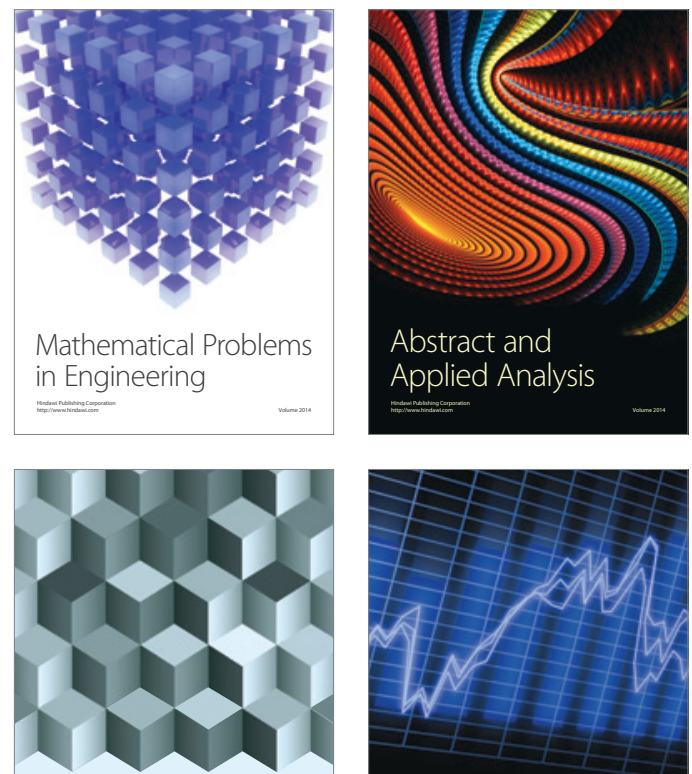

Journal of

Function Spaces

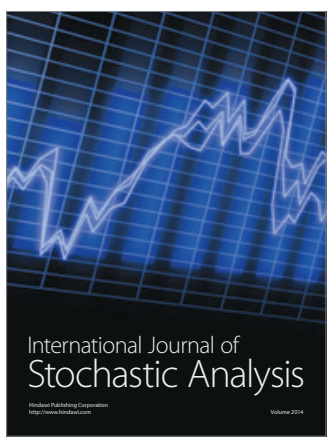

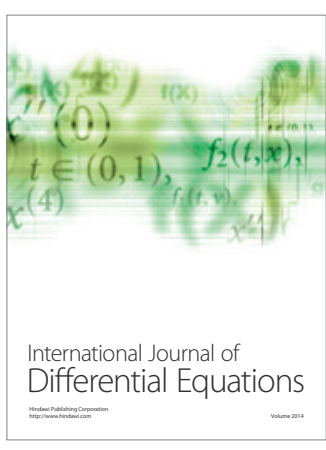
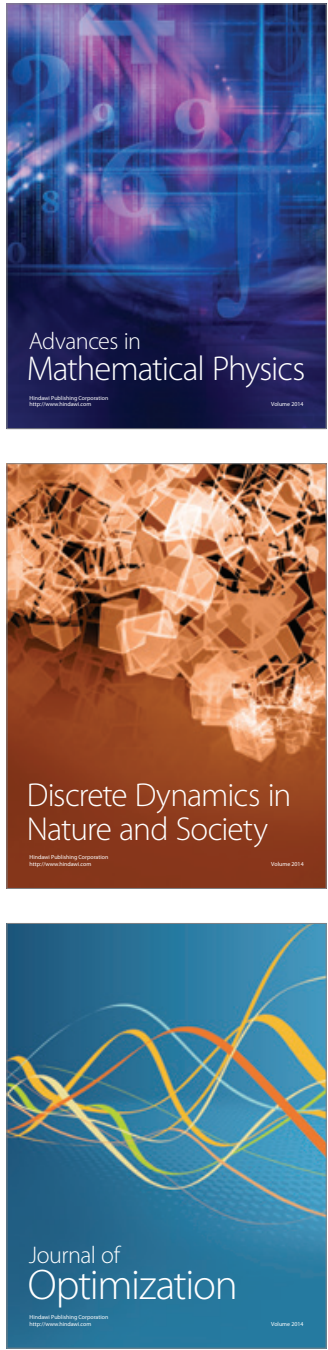